\title{
Anisotropic smoothness classes: from finite element approximation to image models
}

\author{
Jean-Marie Mirebeau and Albert Cohen
}

November 19, 2018

\begin{abstract}
We propose and study quantitative measures of smoothness $f \mapsto A(f)$ which are adapted to anisotropic features such as edges in images or shocks in PDE's. These quantities govern the rate of approximation by adaptive finite elements, when no constraint is imposed on the aspect ratio of the triangles, the simplest example being $A_{p}(f)=\left\|\sqrt{\left|\operatorname{det}\left(d^{2} f\right)\right|}\right\|_{L^{\tau}}$ which appears when approximating in the $L^{p}$ norm by piecewise linear elements when $\frac{1}{\tau}=\frac{1}{p}+1$. The quantities $A(f)$ are not semi-norms, and therefore cannot be used to define linear function spaces. We show that these quantities can be well defined by mollification when $f$ has jump discontinuities along piecewise smooth curves. This motivates for using them in image processing as an alternative to the frequently used total variation semi-norm which does not account for the smoothness of the edges.
\end{abstract}

\section{Introduction}

There exists various ways of measuring the smoothness of functions on a domain $\Omega \subset \mathbb{R}^{\mathrm{d}}$, generally through the definition of an appropriate smoothness space. Classical instances are Sobolev, Hölder and Besov spaces. Such spaces are of common use when describing the regularity of solutions to partial differential equations. From a numerical perspective, they are also useful in order to sharply characterize at which rate a function $f$ may be approximated by simpler functions such as Fourier series, finite elements, splines or wavelets (see [6, 12, 9] for surveys on such results).

Functions arising in concrete applications may have inhomogeneous smoothness properties, in the sense that they exhibit area of smoothness separated by localized discontinuities. Two typical instances are (i) edge in functions representing real images and (ii) shock profiles in solutions to non-linear hyperbolic PDE's. The smoothness space that is best taylored to take such features into account is the space $B V(\Omega)$ of bounded variation functions. This space consists of those $f$ in $L^{1}(\Omega)$ such that $\nabla f$ is a bounded measure, i.e. such that their total variation

$$
\operatorname{TV}(f)=|f|_{B V}:=\max \left\{\int_{\Omega} f \operatorname{div}(\varphi) ; \varphi \in \mathcal{D}(\Omega)^{d},\|\varphi\|_{L^{\infty}} \leq 1\right\}
$$

is finite. Functions of bounded variation are allowed to have jump discontinuities along hypersurfaces of finite measure. In particular, the characteristic function of a smooth subdomain $D \subset \Omega$ has finite total variation equal to the $d$-1-dimensional Hausdorff measure of its boundary:

$$
\left|\chi_{D}\right|_{B V}=\mathcal{H}_{d-1}(\partial D)
$$

It is well known that $B V$ is a regularity space for certain hyperbolic conservation laws [14, 17, in the sense that the total variation of their solutions remains finite for all time $t>0$. This space also plays an important role in image processing since the seminal paper [13. Here, a small total variation is used as a prior to describe the mathematical properties of "plausible images", when trying to restore an unknown image $f$ from an observation $h=T f+e$ where $T$ is a known operator and $e$ a measurement noise of norm $\|e\|_{L^{2}} \leq \varepsilon$. The restored image is then defined as the solution to the minimization problem

$$
\min _{g \in B V}\left\{|g|_{B V} ;\|T g-h\|_{L^{2}} \leq \varepsilon\right\} .
$$


From the point of view of approximation theory, it was shown in 8 that the space $B V$ is almost characterized by expansions in wavelet bases. For example, in dimension $d=2$, if $f=\sum d_{\lambda} \psi_{\lambda}$ is an expansion in a tensor-product $L^{2}$-orthonormal wavelet basis, one has

$$
\left(d_{\lambda}\right) \in \ell^{1} \Rightarrow f \in B V \Rightarrow\left(d_{\lambda}\right) \in w \ell^{1},
$$

where $w \ell^{1}$ is the space of weakly summable sequences. The fact that the wavelet coefficients of a $B V$ function are weakly summable implies the convergence estimate

$$
\left\|f-f_{N}\right\|_{L^{2}} \leq C N^{-1 / 2}|f|_{B V},
$$

where $f_{N}$ is the nonlinear approximation of $f$ obtained by retaining the $N$ largest coefficients in its wavelet expansion. Such approximation results have been further used in order to justify the performance of compression or denoising algorithms based on wavelet thresholding [7].

In recent years, it has been observed that the space $B V$ (and more generally classical smoothness spaces) do not provide a fully satisfactory description of piecewise smooth functions arising in the above mentioned applications. Indeed, formula (1.1) reveals that the total variation only takes into account the size of the sets of discontinuities and not their geometric smoothness. In image processing, this means that the set of bounded variation images does not make the distinction between smooth and non-smooth edges as long as they have finite length.

The fact that edges have some geometric smoothness can be exploited in order to study approximation procedures which outperform wavelet thresholding in terms of convergence rates. For instance, it is easy to prove that if $f=\chi_{D}$ where $D$ is a bidimensional domain with smooth boundary, one can find a sequence of triangulations $\mathcal{T}_{N}$ with $N$ triangles such that the convergence estimate

$$
\left\|f-I_{\mathcal{T}_{N}} f\right\|_{L^{2}} \leq C N^{-1},
$$

holds, where $I_{\mathcal{T}}$ denotes the piecewise linear interpolation operator on a triangulation $\mathcal{T}$. Other methods are based on thresholding a decomposition of the function in bases or frames which differ from classical wavelets, see e.g. [3, 16, 1. These methods also yield improvements over (1.3) similar to (1.4). The common feature in all these approaches is that they achieve anisotropic refinement near the edges. For example, in order to obtain the estimate (1.4), the triangulation $\mathcal{T}_{N}$ should include a thin layer of triangles which approximates the boundary $\partial D$. These triangles typically have size $N^{-2}$ in the normal direction to $\partial D$ and $N^{-1}$ in the tangential direction, and are therefore highly anisotropic.

Intuitively, these methods are well adapted to functions which have anisotropic smoothness properties in the sense that their local variation is significantly stronger in one direction. Such properties are not well described by classical smoothness spaces such as $B V$, and a natural question to ask is therefore:

What type of smoothness properties govern the convergence rate of anisotropic refinement methods and how can one quantify these properties?

The goal of this paper is to answer this question, by proposing and studying measures of smoothness which are suggested by recent results on anisotropic finite element approximation [2, 5]. Before going further, let us mention several existing approaches which have been developed for describing and quantifying anisotropic smoothness, and explain their limitations.

1. The so-called mixed smoothness classes have been introduced and studied in order to describe functions which have a different order of smoothness in each coordinate, see e.g. [20, 23. These spaces are therefore not adapted to our present goal since the anisotropic smoothness that we want to describe may have preferred directions that are not aligned with the coordinate axes and that may vary from one point to another (for example an image with a curved edge).

2. Anisotropic smoothness spaces with more general and locally varying directions have been investigated in [15. Yet, in such spaces the amount of smoothness in different directions at each point is still fixed in advance and therefore again not adapted to our goal, since this amount may differ from one function to another (for example two images with edges located at different positions). 
3. A class of functions which is often used to study the convergence properties of anisotropic approximation methods is the family of $C^{m}-C^{n}$ cartoon images, i.e. functions which are $C^{m}$ smooth on a finite number of subdomains $\left(\Omega_{i}\right)_{i=1, \cdots, k}$ separated by a union of discontinuity curves $\left(\Gamma_{j}\right)_{j=1, \cdots, l}$ that are $C^{n}$ smooth. The defects of this class are revealed when searching for simple expression that quantifies the amount of smoothness in this sense. A natural choice is to take the supremum of all $C^{m}\left(\Omega_{i}\right)$ norms of $f$ and $C^{n}$ norms of the normal parametrization of $\Gamma_{j}$. We then observe that this quantity is unstable in the sense that it becomes extremely large for blurry images obtained by convolving a cartoon image by a mollifier $\varphi_{\delta}=\frac{1}{\delta^{2}} \varphi(\dot{\bar{h}})$ as $\delta \rightarrow 0$. In addition, this quantity does not control the number of subdomains in the partition.

4. A recent approach proposed in [10 defines anisotropic smoothness through the geometric smoothness properties of the level sets of the function $f$. In this approach the measure of smoothness is not simple to compute directly from $f$ since it involves each of its level sets and a smoothness measure of their local parametrization.

The results of [2, 5, 19] describe the $L^{p}$-error of piecewise linear interpolation by an optimally adapted triangulation of at most $N$ elements, when $f$ is a $C^{2}$ function of two variables. This error is defined as

$$
\sigma_{N}(f)_{p}:=\inf _{\#(\mathcal{T}) \leq N}\left\|f-I_{\mathcal{T}} f\right\|_{L^{p}} .
$$

It is shown in [2] for $p=\infty$ and in [19] for all $1 \leq p \leq \infty$ that that

$$
\limsup _{N \rightarrow+\infty} N \sigma_{N}(f)_{p} \leq C A_{p}(f),
$$

where $C$ is an absolute constant and

$$
A_{p}(f):=\left\|\sqrt{\left|\operatorname{det}\left(d^{2} f\right)\right|}\right\|_{L^{\tau}}, \frac{1}{\tau}:=1+\frac{1}{p} .
$$

Moreover, this estimate is known to be optimal in the sense that $\liminf _{N \rightarrow+\infty} N \sigma_{N}(f)_{p} \geq c A_{p}(f)$ also holds, under some mild restriction on the class of triangulations in which one selects the optimal one. These results are extended in [19] to the case of higher order finite elements and space dimension $d>2$, for which one can identify similar measures $f \mapsto A(f)$ governing the convergence estimate. Such quantities thus constitute natural candidates to measure anisotropic smoothness properties. Note that $A_{p}(f)$ is not a semi-norm due to the presence of the determinant in (1.6), and in particular the quasi-triangle inequality $A_{p}(f+g) \leq C\left(A_{p}(f)+A_{p}(g)\right)$ does not hold even with $C>1$.

Our paper is organized as follows. We begin in $\S 2$ by a brief account of the available estimates on anisotropic finite element interpolation, and we recall in particular the argument that leads to (1.5) with the quantity $A_{p}(f)$ defined by (1.6).

Since $A_{p}(f)$ is not a norm, we cannot associate a linear smoothness space to it by a standard completion process. We are thus facing a difficulty in extending the definition of $A_{p}(f)$ to functions which are not $C^{2}$-smooth and in particular to cartoon images such as in item 3 above. Since we know from (1.4) that for such cartoon images the $L^{2}$ error of adaptive piecewise linear interpolation decays like $N^{-1}$, we would expect that the quantity

$$
A_{2}(f)=\left\|\sqrt{\left|\operatorname{det}\left(d^{2} f\right)\right|}\right\|_{L^{2 / 3}},
$$

corresponding to the case $p=2$ can be properly defined for piecewise smooth functions. We address this difficulty in $\S 3$ by a regularization process: if $f$ is a cartoon image we introduce its regularized version

$$
f_{\delta}:=f * \varphi_{\delta},
$$

where $\varphi_{\delta}=\frac{1}{\delta^{2}} \varphi(\dot{\bar{h}})$ is a standard mollifier. Our main result is the following: for any cartoon image $f$ of $C^{2}-C^{2}$ type, the quantity $A_{2}\left(f_{\delta}\right)$ remains uniformly bounded as $\delta \rightarrow 0$ and one has

$$
\lim _{\delta \rightarrow 0} A_{2}\left(f_{\delta}\right)^{2 / 3}=\sum_{i=1}^{k} \int_{\Omega_{i}}\left|\sqrt{\left|\operatorname{det}\left(d^{2} f\right)\right|}\right|^{2 / 3}+C(\varphi) \sum_{j=1}^{l} \int_{\Gamma_{j}}|[f](s)|^{2 / 3}|\kappa(s)|^{1 / 3} d s,
$$


where $[f](s)$ and $\kappa(s)$ respectively denote the jump of $f$ and the curvature of $\Gamma_{j}$ at the point $s$, and where $C(\varphi)$ is a constant that only depends on the choice of the mollifier. This constant can be shown to be uniformly bounded by below for the class of radially decreasing mollifiers. This result reveals that $A_{2}(f)$ is stable under regularization of cartoon images (in contrast to the measure of smoothness described in item 3 above). We also discuss the behaviour of $A_{p}$ when $p \neq 2$.

These results lead us in $\S 4$ to a comparison between the quantity $A_{2}(f)$ and the total variation TV $(f)$. We also make some remarks on the existing links between the limit expression in (1.8) and classical results on adaptive approximation of curves, as well as with operators of affine-invariant image processing which also involve the power $1 / 3$ of the curvature.

We devote $\S 5$ to numerical tests performed on cartoon images that illustrate the validity of our results and we describe in $\S 6$ the extension of our results to finite elements of higher degree and higher space dimensions. Concluding remarks and perspectives of our work are given in $\S 7$.

\section{Anisotropic finite element approximation}

A standard estimate in finite element approximation states that if $f \in W^{2, p}(\Omega)$ then

$$
\left\|f-I_{\mathcal{T}_{h}} f\right\|_{L^{p}} \leq C h^{2}\left\|d^{2} f\right\|_{L^{p}}
$$

where $\mathcal{T}_{h}$ is a triangulation of mesh size $h:=\max _{T \in \mathcal{T}_{h}} \operatorname{diam}(T)$. If we restrict our attention to a family quasi-uniform triangulations, $h$ is linked with the complexity $N:=\#\left(\mathcal{T}_{h}\right)$ according to

$$
C_{1} h^{-2} \leq N \leq C_{2} h^{-2}
$$

Therefore, denoting by $\sigma_{N}^{\text {unif }}(f)_{L^{p}}$ the $L^{p}$ approximation by quasi-uniform triangulations of cardinality $N$, we can re-express the above estimate as

$$
\sigma_{N}^{\text {unif }}(f)_{L^{p}} \leq C N^{-1}\left\|d^{2} f\right\|_{L^{p}} .
$$

In order to explain how this estimate can be improved when using adaptive partitions, we first give some heuristic arguments which are based on the assumption that on each triangle $T$ the relative variation of $d^{2} f$ is small so that it can be considered as constant over $T$, which means that $f$ coincides with a quadratic function $q_{T}$ on each $T$. Denoting by $I_{T}$ the local interpolation operator on a triangle $T$ and by $e_{T}(f)_{p}:=\left\|f-I_{T} f\right\|_{L^{p}(T)}$ the local $L^{p}$ error, we thus have according to this heuristics

$$
\left\|f-I_{\mathcal{T}} f\right\|_{L^{p}}=\left(\sum_{T \in \mathcal{T}} e_{T}(f)_{p}^{p}\right)^{\frac{1}{p}}=\left(\sum_{T \in \mathcal{T}} e_{T}\left(q_{T}\right)_{p}^{p}\right)^{\frac{1}{p}}
$$

We are thus led to study the local interpolation error $e_{T}(q)_{p}$ when $q \in \mathbb{P}_{2}$ is a a quadratic polynomial. Denoting by $\mathbf{q}$ the homogeneous part of $q$, we remark that

$$
e_{T}(q)_{p}=e_{T}(\mathbf{q})_{p} .
$$

We optimize the shape of $T$ with respect to the quadratic form $\mathbf{q}$ by introducing a function $K_{p}$ defined on the space of quadratic forms by

$$
K_{p}(\mathbf{q}):=\inf _{|T|=1} e_{T}(\mathbf{q})_{p},
$$

where the infimum is taken among all triangles of area 1 . It is easily seen that $e_{T}(\mathbf{q})_{p}$ is invariant by translation of $T$ and so is therefore the minimizing triangle if it exists. By homogeneity, it is also easily seen that

$$
\inf _{|T|=a} e_{T}(\mathbf{q})_{p}=a^{\frac{1}{\tau}} K_{p}(\mathbf{q}), \frac{1}{\tau}:=\frac{1}{p}+1,
$$

and that the minimizing triangle of area $a$ is obtained by rescaling the minimizing triangle of area 1 if it exists. Finally, it is easily seen that if $\varphi$ is an invertible linear transform

$$
K_{p}(\mathbf{q} \circ \varphi)=|\operatorname{det}(\varphi)| K_{p}(\mathbf{q}),
$$


and that the minimizing triangle of area $|\operatorname{det}(\varphi)|^{-1}$ for $\mathbf{q} \circ \varphi$ is obtained by application of $\varphi^{-1}$ to the minimizing triangle of area 1 for $\mathbf{q}$ if it exists. If $\operatorname{det}(\mathbf{q}) \neq 0$, there exists a $\varphi$ such that $\mathbf{q} \circ \varphi$ is either $x^{2}+y^{2}$ or $x^{2}-y^{2}$ up to a sign change, and we have $|\operatorname{det}(\mathbf{q})|=|\operatorname{det}(\varphi)|^{-2}$. It follows that $K_{p}(\mathbf{q})$ has the simple form

$$
K_{p}(\mathbf{q})=\sigma|\operatorname{det}(\mathbf{q})|^{1 / 2},
$$

where $\sigma$ is a constant equal to $K_{p}\left(x^{2}+y^{2}\right)$ if $\operatorname{det}(\mathbf{q})>0$ and to $K_{p}\left(x^{2}-y^{2}\right)$ if $\operatorname{det}(\mathbf{q})<0$. One easily checks that this equality also holds when $\operatorname{det}(\mathbf{q})=0$ in which case $K_{p}(\mathbf{q})=0$.

Assuming that the triangulation $\mathcal{T}$ is such that all its triangles $T$ have optimized shape in the above sense with respect to the quadratic form $\mathbf{q}_{T}$ associated with $q_{T}$, we thus have for any triangle $T \in \mathcal{T}$

$$
e_{T}(f)_{p}=e_{T}\left(\mathbf{q}_{T}\right)_{p}=|T|^{\frac{1}{\tau}} K_{p}\left(\mathbf{q}_{T}\right)=\left\|K_{p}\left(\frac{d^{2} f}{2}\right)\right\|_{L^{\tau}(T)} .
$$

since we have assumed $\frac{d^{2} f}{2}=\mathbf{q}_{T}$ on $T$. In order to optimize the trade-off between the global error and the complexity $N=\#(\mathcal{T})$, we apply the principle of error equidistribution: the triangles $T$ have area such that all errors $e_{T}\left(\mathbf{q}_{T}\right)_{p}$ are equal i.e. $e_{T}\left(\mathbf{q}_{T}\right)_{p}=\eta$ for some $\eta>0$ independent of $T$. It follows that

$$
N \eta^{\tau} \leq\left\|K_{p}\left(\frac{d^{2} f}{2}\right)\right\|_{L^{\tau}(\Omega)}^{\tau},
$$

and therefore

$$
\sigma_{N}(f)_{p} \leq\left\|f-I_{\mathcal{T}} f\right\|_{L^{p}} \leq N^{1 / p} \eta \leq\left\|K_{p}\left(\frac{d^{2} f}{2}\right)\right\|_{L^{\tau}(\Omega)} N^{-1}
$$

which according to (2.10) implies

$$
\sigma_{N}(f)_{p} \leq C N^{-1} A_{p}(f),
$$

with $A_{p}$ defined as in (1.6).

The estimate (2.11) is too optimistic to be correct: if $f$ is a univariate function then $A_{p}(f)=0$ while $\sigma_{N}(f)_{p}$ may not vanish. In a rigorous derivation such as in [2], one observes that if $f \in C^{2}$, the replacement of $d^{2} f$ by a constant over $T$ induces an error which becomes negligible only when the triangles are sufficiently small, and therefore a correct statement is that for any $\varepsilon>0$ there exists $N_{0}=N_{0}(f, \varepsilon)$ such that

$$
\sigma_{N}(f)_{p} \leq N^{-1}\left(\left\|K_{p}\left(\frac{d^{2} f}{2}\right)\right\|_{L^{\tau}(\Omega)}+\varepsilon\right),
$$

for all $N \geq N_{0}$, i.e.

$$
\limsup _{N \rightarrow+\infty} N \sigma_{N}(f)_{p} \leq\left\|K_{p}\left(\frac{d^{2} f}{2}\right)\right\|_{L^{\tau}(\Omega)}
$$

which according to (2.10) implies (1.5).

\section{$3 \quad$ Piecewise smooth functions and images}

As already observed, the quantities $A_{p}(f)$ are well defined for functions $f \in C^{2}$, but we expect that they should in some sense also be well defined for functions representing $C^{2}-C^{2}$ "cartoon images" when $p \leq 2$. We first give a precise definition of such functions.

Definition 3.1 A cartoon function on an open set $\Omega$ is a function almost everywhere of the form

$$
f=\sum_{1 \leq i \leq k} f_{i} \chi_{\Omega_{i}}
$$

where the $\Omega_{i}$ are disjoint open sets with piecewise $C^{2}$ boundary, no cusps (i.e. satisfying an interior and exterior cone condition), and such that $\bar{\Omega}=\cup_{i=1}^{k} \bar{\Omega}_{i}$, and where for each $1 \leq i \leq k$, the function $f_{i}$ is $C^{2}$ on $\bar{\Omega}_{i}$. 
Let us consider a fixed cartoon function $f$ on an open polygonal domain $\Omega$ (i.e. $\Omega$ is such that $\bar{\Omega}$ is a closed polygon) associated with a decomposition $\left(\Omega_{i}\right)_{1 \leq i \leq k}$. We define

$$
\Gamma:=\bigcup_{1 \leq i \leq k} \partial \Omega_{i}
$$

the union of the boundaries of the $\Omega_{i}$. The above definition implies that $\Gamma$ is the disjoint union of a finite set of points $\mathcal{P}$ and a finite number of open curves $\left(\Gamma_{i}\right)_{1 \leq i \leq l}$.

$$
\Gamma=\left(\bigcup_{1 \leq i \leq l} \Gamma_{i}\right) \cup \mathcal{P}
$$

Furthermore for all $1 \leq i<j \leq l$, we may impose that $\bar{\Gamma}_{i} \cap \bar{\Gamma}_{j} \subset \mathcal{P}$ (this may be ensured by a splitting of some of the $\Gamma_{i}$ if necessary).

We now consider the piecewise linear interpolation $I_{\mathcal{T}_{N}} f$ of $f$ on a triangulation $\mathcal{T}_{N}$ of cardinality $N$. We distinguish two types of elements of $\mathcal{T}_{N}$. A triangle $T \in \mathcal{T}_{N}$ is called "regular" if $T \cap \Gamma=\emptyset$, and we denote the set of such triangles by $\mathcal{T}_{N}^{r}$. Other triangles are called "edgy" and their set is denoted by $\mathcal{T}_{N}^{e}$. We can thus split $\Omega$ according to

$$
\Omega:=\left(\cup_{T \in \mathcal{T}_{N}^{r}} T\right) \cup\left(\cup_{T \in \mathcal{T}_{N}^{e}} T\right)=\Omega_{N}^{r} \cup \Omega_{N}^{e} .
$$

We split accordingly the $L^{p}$ interpolation error into

$$
\left\|f-I_{\mathcal{T}_{N}} f\right\|_{L^{p}(\Omega)}^{p}=\int_{\Omega_{N}^{r}}\left|f-I_{\mathcal{T}_{N}} f\right|^{p}+\int_{\Omega_{N}^{e}}\left|f-I_{\mathcal{T}_{N}} f\right|^{p} .
$$

We may use $\mathcal{O}(N)$ triangles in $\mathcal{T}_{N}^{e}$ and $\mathcal{T}_{N}^{r}$ (for example $N / 2$ in each set). Since $f$ has discontinuities along $\Gamma$, the $L^{\infty}$ interpolation error on $\Omega_{N}^{e}$ does not tend to zero and $\mathcal{T}_{N}^{e}$ should be chosen so that $\Omega_{N}^{e}$ has the aspect of a thin layer around $\Gamma$. Since $\Gamma$ is a finite union of $C^{2}$ curves, we can build this layer of width $\mathcal{O}\left(N^{-2}\right)$ and therefore of global area $\left|\Omega_{N}^{e}\right| \leq C N^{-2}$, by choosing long and thin triangles in $\mathcal{T}_{N}^{e}$. On the other hand, since $f$ is uniformly $C^{2}$ on $\Omega_{N}^{r}$, we may choose all triangles in $\mathcal{T}_{N}^{r}$ of regular shape and diameter $h_{T} \leq C N^{-1 / 2}$. Hence we obtain the following heuristic error estimate, for a well designed anisotropic triangulation:

$$
\begin{aligned}
\left\|f-I_{\mathcal{T}_{N}} f\right\|_{L^{p}(\Omega)} & =\left(\left\|f-I_{\mathcal{T}_{N}} f\right\|_{L^{p}\left(\Omega_{N}^{r}\right)}^{p}+\left\|f-I_{\mathcal{T}_{N}} f\right\|_{L^{p}\left(\Omega_{N}^{e}\right)}^{p}\right)^{1 / p} \\
& \leq\left(\left\|f-I_{\mathcal{T}_{N}} f\right\|_{L^{\infty}\left(\Omega_{N}^{r}\right)}^{p}\left|\Omega_{N}^{r}\right|+\left\|f-I_{\mathcal{T}_{N}} f\right\|_{L^{\infty}\left(\Omega_{N}^{e}\right.}^{p}\left|\Omega_{N}^{e}\right|\right)^{1 / p} \\
& \leq C\left(N^{-p}+N^{-2}\right)^{1 / p}
\end{aligned}
$$

and therefore

$$
\left\|f-I_{\mathcal{T}_{N}} f\right\|_{L^{p}(\Omega)} \leq C N^{-\min \{1,2 / p\}}
$$

where the constant $C$ depends on $\left\|d^{2} f\right\|_{L^{\infty}(\Omega \backslash \Gamma)},\|f\|_{L^{\infty}(\Omega)}$ and on the number, length and maximal curvature of the $C^{2}$ curves which constitute $\Gamma$.

Observe in particular that the error is dominated by the edge term $\left\|f-I_{\mathcal{T}_{N}} f\right\|_{L^{p}\left(\Omega_{N}^{e}\right)}$ when $p>2$ and by the smooth term $\left\|f-I_{\mathcal{T}_{N}} f\right\|_{L^{p}\left(\Omega_{N}^{r}\right)}$ when $p<2$. For the critical value $p=2$ the two terms have the same order.

For $p \leq 2$, we obtain the approximation rate $N^{-1}$ which suggests that approximation results such as (1.5) should also apply to cartoon functions and that the quantity $A_{p}(f)$ should be finite. We would therefore like to bridge the gap between anisotropic approximation of cartoon functions and smooth functions. For this purpose, we first need to give a proper meaning to $A_{p}(f)$ when $f$ is a cartoon function. This is not straightforward, due to the fact that the product of two distributions has no meaning in general. Therefore, we cannot $\operatorname{define} \operatorname{det}\left(d^{2} f\right)$ in a distributional sense, when the coefficients of $d^{2} f$ are distributions without sufficient smoothness. Our approach will rather be based on regularisation. This is additionally justified by the fact that sharp curves of discontinuity are a mathematical idealisation. In 
real world applications, such as photography, several physical limitations (depth of field, optical blurring) impose a certain level of blur on the edges.

In the following, we consider a fixed radial nonnegative function $\varphi$ of unit integral and supported in the unit ball, and we define for all $\delta>0$ and $f$ defined on $\Omega$,

$$
\varphi_{\delta}(z):=\frac{1}{\delta^{2}} \varphi\left(\frac{z}{\delta}\right) \text { and } f_{\delta}=f * \varphi_{\delta} .
$$

Our main result gives a meaning to $A_{p}(f)$ based on this regularization. If $f$ is a cartoon function on a set $\Omega$, and if $x \in \Gamma \backslash \mathcal{P}$, we denote by $[f](x)$ the jump of $f$ at this point. We also denote $\mathbf{t}(x)$ and $\mathbf{n}(x)$ the unit tangent and normal vectors to $\Gamma$ at $x$ oriented in such way that $\operatorname{det}(\mathbf{t}, \mathbf{n})=+1$, and by $\kappa(x)$ the curvature at $x$ which is defined by the relation

$$
\partial_{\mathbf{t}(x)} \mathbf{t}(x)=\kappa(x) \mathbf{n}(x) .
$$

For $p \in[1, \infty]$ and $\tau$ defined by $\frac{1}{\tau}:=1+\frac{1}{p}$, we introduce the two quantities

$$
\begin{aligned}
S_{p}(f) & :=\left\|\sqrt{\left|\operatorname{det}\left(d^{2} f\right)\right|}\right\|_{L^{\tau}(\Omega \backslash \Gamma)}=A_{p}\left(f_{\mid \Omega \backslash \Gamma}\right), \\
E_{p}(f) & :=\|\sqrt{|\kappa|}[f]\|_{L^{\tau}(\Gamma)},
\end{aligned}
$$

which respectively measure the "smooth part" and the "edge part" of $f$. We also introduce the constant

$$
C_{p, \varphi}:=\left\|\sqrt{\left|\Phi \Phi^{\prime}\right|}\right\|_{L^{\tau}(\mathbb{R})}, \quad \text { where } \Phi(x):=\int_{y \in \mathbb{R}} \varphi(x, y) d y .
$$

Note that $f_{\delta}$ is only properly defined on the set

$$
\Omega^{\delta}:=\{z \in \Omega ; B(z, \delta) \subset \Omega\},
$$

and therefore, we define $A_{p}\left(f_{\delta}\right)$ as the $L^{\tau}$ norm of $\sqrt{\left|\operatorname{det}\left(d^{2} f_{\delta}\right)\right|}$ on this set.

Theorem 3.2 For all cartoon functions $f$, the quantity $A_{p}\left(f_{\delta}\right)$ behaves as follows:

- If $p<2$, then

$$
\lim _{\delta \rightarrow 0} A_{p}\left(f_{\delta}\right)=S_{p}(f)
$$

- If $p=2$, then $\tau=\frac{2}{3}$ and

$$
\lim _{\delta \rightarrow 0} A_{2}\left(f_{\delta}\right)=\left(S_{2}(f)^{\tau}+E_{2}(f)^{\tau} C_{2, \varphi}^{\tau}\right)^{\frac{1}{\tau}} .
$$

- If $p>2$, then $A_{p}\left(f_{\delta}\right) \rightarrow \infty$ according to

$$
\lim _{\delta \rightarrow 0} \delta^{\frac{1}{2}-\frac{1}{p}} A_{p}\left(f_{\delta}\right)=E_{p}(f) C_{p, \varphi}
$$

Remark 3.3 This theorem reveals that as $\delta \rightarrow 0$, the contribution of the neighbourhood of $\Gamma$ to $A_{p}\left(f_{\delta}\right)$ is negligible when $p<2$ and dominant when $p>2$, which was already remarked in the heuristic computation leading to (3.15).

Remark 3.4 It seems to be possible to eliminate the "no cusps" condition in the definition of cartoon functions, while still retaining the validity of this theorem. It also seems possible to take the more natural choice $\varphi(z)=\frac{1}{\pi} e^{-\|z\|^{2}}$, which is not compactly supported. However, both require higher technicality in the proof which we avoid here.

Before attacking the proof of Theorem 3.2, we show below that the constant $C_{p, \varphi}$ involved in the result for $p \geq 2$ is uniformly bounded by below for a mild class of mollifiers. 
Proposition 3.5 Let $\varphi$ be a radial and positive function supported on the unit ball such that $\int \varphi=1$ and that $\varphi(x)$ decreases as $|x|$ increases. For any $p \geq 2$ we have

$$
C_{p, \varphi} \geq \frac{2}{\pi}\left(\frac{4}{\tau+2}\right)^{\frac{1}{\tau}}
$$

and this lower bound is optimal. There is no such bound if $p<2$, but note that Theorem 3.2 does not involve $C_{p, \varphi}$ for $p<2$.

Proof: Let $D$ be the unit disc of $\mathbb{R}^{2}$. We define a non smooth mollifier $\psi$ and a function $\Psi$ as follows

$$
\psi:=\frac{\chi_{D}}{\pi} \text { and } \Psi(x):=\int_{\mathbb{R}} \psi(x, y) d y .
$$

One easily obtains that $\Psi(x)=\frac{2}{\pi} \sqrt{1-x^{2}} \chi_{[-1,1]}(x)$ and $\Psi^{\prime}(x)=\frac{-2 x}{\pi \sqrt{1-x^{2}}} \chi_{[-1,1]}(x)$, hence

$$
\Psi(x) \Psi^{\prime}(x)=\frac{-4 x}{\pi^{2}} \chi_{[-1,1]}(x) .
$$

For all $\delta>0$ we define $\psi_{\delta}:=\delta^{-2} \psi\left(\delta^{-1} \cdot\right)$, and $\Psi_{\delta}(x):=\int_{\mathbb{R}} \psi_{\delta}(x, y) d y$. Similarly we obtain

$$
\Psi_{\delta}(x) \Psi_{\delta}^{\prime}(x)=\frac{-4 x}{\pi^{2}} \chi_{[-\delta, \delta]} \delta^{-4} .
$$

Hence

$$
C_{p, \psi_{\delta}}=\left\|\sqrt{\Psi_{\delta}(x)\left|\Psi_{\delta}^{\prime}(x)\right|}\right\|_{L^{\tau}(\mathbb{R})}=\frac{2}{\pi \delta^{2}}\left(\int_{-\delta}^{\delta}|x|^{\frac{\tau}{2}} d x\right)^{\frac{1}{\tau}}=\frac{2}{\pi \delta^{2}}\left(\frac{2 \delta^{\frac{\tau}{2}+1}}{\frac{\tau}{2}+1}\right)^{\frac{1}{\tau}}=\frac{2}{\pi}\left(\frac{4}{\tau+2}\right)^{\frac{1}{\tau}} \delta^{\frac{1}{p}-\frac{1}{2}} .
$$

Note that

$$
\text { If } p \geq 2 \text { and } \delta \in(0,1] \text { then } C_{p, \psi_{\delta}} \geq C_{p, \psi}=\frac{2}{\pi}\left(\frac{4}{\tau+2}\right)^{\frac{1}{\tau}} \text {. }
$$

The mollifier $\varphi$ of interest is radially decreasing, has unit integral and is supported on the unit ball. It follows that there exists a Lebesgue measure $\mu$ on $(0,1]$, of mass 1 , such that

$$
\varphi=\int_{0}^{1} \psi_{\delta} d \mu(\delta)
$$

Hence $\Phi(x):=\int_{\mathbb{R}} \varphi(x, y) d y=\int_{0}^{1} \Psi_{\delta}(x) d \mu(\delta)$, for any $x \in \mathbb{R}$. Since $s \mapsto s^{\tau}$ is concave on $\mathbb{R}_{+}$when $0<\tau \leq 1$, we obtain

$$
\Phi(x)^{\tau}=\left(\int_{0}^{1} \Psi_{\delta}(x) d \mu(\delta)\right)^{\tau} \geq \int_{0}^{1} \Psi_{\delta}(x)^{\tau} d \mu(\delta)
$$

Similarly, since the sign of $\Psi_{\delta}^{\prime}(x)$ is independent of $\delta,\left|\Phi^{\prime}(x)\right|^{\tau}=\left(\int_{0}^{1}\left|\Psi_{\delta}^{\prime}(x)\right| d \mu(\delta)\right)^{\tau} \geq \int_{0}^{1}\left|\Psi_{\delta}^{\prime}(x)\right|^{\tau} d \mu(\delta)$. Applying the Cauchy-Schwartz inequality we obtain

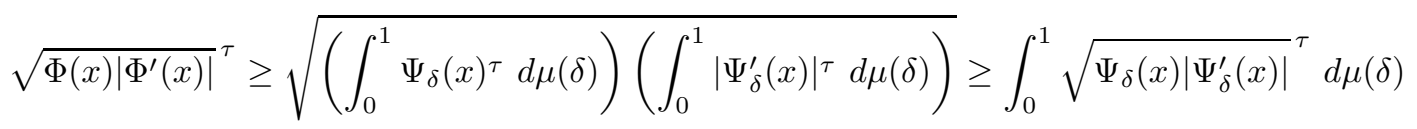

Eventually we conclude using the previous equation and (3.20) as follows

$$
C_{p, \varphi}^{\tau}=\int_{\mathbb{R}}{\sqrt{\Phi\left|\Phi^{\prime}\right|}}^{\tau} \geq \int_{0}^{1}\left(\int_{\mathbb{R}}{\sqrt{\Psi_{\delta}\left|\Psi_{\delta}^{\prime}\right|}}^{\tau}\right) d \mu(\delta)=\int_{0}^{1} C_{p, \psi_{\delta}}^{\tau} d \mu(\delta) \geq C_{p, \psi}^{\tau},
$$

which concludes the proof of this lemma. 
The rest of this section is devoted to the proof of Theorem 3.2. Since it is rather involved, we split its presentation into several main steps.

Step 1: decomposition of $A_{p}\left(f_{\delta}\right)$. Using the notation $K(M):=\sqrt{|\operatorname{det} M|}$, we can write

$$
A_{p}\left(f_{\delta}\right)^{\tau}=\int_{\Omega^{\delta}} K\left(d^{2} f_{\delta}\right)^{\tau}
$$

We decompose this quantity based on a partition of $\Omega^{\delta}$ into three subsets

$$
\Omega^{\delta}=\Omega_{\delta} \cup \Gamma_{\delta} \cup \mathcal{P}_{\delta}
$$

The first set $\Omega_{\delta}$ corresponds to the smooth part:

$$
\Omega_{\delta}:=\bigcup_{1 \leq i \leq k} \Omega_{i, \delta}, \text { where } \Omega_{i, \delta}:=\left\{z \in \Omega_{i} ; d\left(z, \Omega \backslash \Omega_{i}\right)>\delta\right\} .
$$

Note that $\Omega_{\delta}$ is strictly contained in $\Omega^{\delta}$. The second set corresponds to the edge part: we first define

$$
\Gamma_{\delta}^{0}:=\bigcup_{1 \leq j \leq l} \Gamma_{j, \delta}^{0}, \quad \text { where } \Gamma_{j, \delta}^{0}:=\left\{z \in \Gamma_{j} ; d\left(z, \Gamma \backslash \Gamma_{j}\right)>2 \delta\right\},
$$

and then set

$$
\Gamma_{\delta}:=\bigcup_{1 \leq j \leq l} \Gamma_{j, \delta} \text { where } \Gamma_{j, \delta}:=\left\{z \in \Omega ; d(z, \Gamma)<\delta \text { and } \pi_{\Gamma}(z) \in \Gamma_{j, \delta}^{0}\right\}
$$

where $\pi_{\Gamma}(z)$ denotes the point of $\Gamma$ which is the closest to $z$. The third set corresponds the corner part:

$$
\mathcal{P}_{\delta}:=\Omega^{\delta} \backslash\left(\Omega_{\delta} \cup \Gamma_{\delta}\right)
$$

The measures of the sets $\Gamma_{\delta}$ and $\mathcal{P}_{\delta}$ tends to 0 as $\delta \rightarrow 0$, while $\left|\Omega_{\delta}\right|$ tends to $|\Omega|$. More precisely, we have

$$
\left|\Gamma_{\delta}\right| \leq C \delta \text { and }\left|\mathcal{P}_{\delta}\right| \leq C \delta^{2}
$$

where the last estimate exploits the "no cusps" property of the cartoon function. We analyze separately the contributions of these three sets to (3.21).

Step 2: Contribution of the smooth part $\Omega_{\delta}$. The contribution of $\Omega_{\delta}$ to the integral (3.21) is easily measured. Indeed, let us define

$$
Q_{\delta}(z):=\left\{\begin{array}{cc}
K\left(d^{2} f_{\delta}(z)\right)^{\tau} & \text { if } z \in \Omega_{\delta} \\
0 & \text { otherwise }
\end{array}\right.
$$

then we have pointwise convergence $Q_{\delta}(z) \rightarrow K\left(d^{2} f(z)\right)^{\tau}$ on $\Omega \backslash \Gamma$. Since the $\delta$-neighbourhood of $\Omega_{\delta}$ is included in $\Omega \backslash \Gamma$, we have

$$
\left\|d^{2}\left(f * \varphi_{\delta}\right)\right\|_{L^{\infty}\left(\Omega_{\delta}\right)}=\left\|\left(d^{2} f\right) * \varphi_{\delta}\right\|_{L^{\infty}\left(\Omega_{\delta}\right)} \leq\left\|d^{2} f\right\|_{L^{\infty}(\Omega \backslash \Gamma)}\left\|\varphi_{\delta}\right\|_{L^{1}}=\left\|d^{2} f\right\|_{L^{\infty}(\Omega \backslash \Gamma)}
$$

Since $K$ is 1 -homogeneous and continuous, we have

$$
K\left(d^{2} f_{\delta}\right) \leq C_{K}\left\|d^{2} f\right\|_{L^{\infty}(\Omega \backslash \Gamma)}, \quad C_{K}:=\max _{\|M\|=1} K(M),
$$

and we conclude by dominated convergence that

$$
\lim _{\delta \rightarrow 0} \int_{\Omega_{\delta}} K\left(d^{2} f_{\delta}\right)^{\tau}=\lim _{\delta \rightarrow 0} \int_{\Omega \backslash \Gamma} Q_{\delta}=\int_{\Omega \backslash \Gamma} K\left(d^{2} f\right)^{\tau} .
$$

Step 3: Contribution of the corner part $\mathcal{P}_{\delta}$. We only need a rough upper estimate of the contribution of $\mathcal{P}_{\delta}$ to the integral (3.21). We observe that

$$
\left\|d^{2}\left(f * \varphi_{\delta}\right)\right\|_{L^{\infty}(\Omega)}=\left\|f *\left(d^{2} \varphi_{\delta}\right)\right\|_{L^{\infty}(\Omega)} \leq\|f\|_{L^{\infty}(\Omega)}\left\|d^{2} \varphi_{\delta}\right\|_{L^{1}\left(\mathbb{R}^{2}\right)}=\frac{M}{\delta^{2}},
$$


where $M:=\|f\|_{L^{\infty}(\Omega)}\left\|d^{2} \varphi\right\|_{L^{1}\left(\mathbb{R}^{2}\right)}$. It follows that

$$
\int_{\mathcal{P}_{\delta}} K\left(d^{2} f_{\delta}\right)^{\tau} \leq\left|\mathcal{P}_{\delta}\right|\left(C_{K}\left(\frac{M}{\delta^{2}}\right)\right)^{\tau} \leq C \delta^{2-2 \tau}
$$

If $\tau<1$, this quantity tends to 0 and is therefore negligible compared to the contribution of the smooth part. If $\tau=1$, which corresponds to $p=\infty$, our further analysis shows that the contribution of the edge part tends to $+\infty$, and therefore the contribution of the corner part is always negligible.

Step 4: Contribution of the edge part $\Gamma_{\delta}$. This step is the main difficulty of the proof. We make a key use of an asymptotic analysis of $f_{\delta}$ on $\Gamma_{\delta}$, which relates its second derivatives to the jump $[f]$ and the curvature $\kappa$ as $\delta \rightarrow 0$. We first define for all $\delta>0$ the map

$$
\begin{array}{ccc}
U_{\delta}: \Gamma \backslash \mathcal{P} \times[-1,1] & \rightarrow & \Omega \\
(x, u) & \mapsto & x+\delta u \mathbf{n}(x) .
\end{array}
$$

We notice that according to our definitions, for $\delta$ small enough, the map $U_{\delta}$ induces a diffeomorphism between $\Gamma_{\delta}^{0} \times[-1,1]$ and $\Gamma_{\delta}$, such that $\pi_{\Gamma}\left(U_{\delta}(x, u)\right)=x$ and $d\left(U_{\delta}(x, u), \Gamma\right)=\left|U_{\delta}(x, u)-x\right|=\delta|u|$. We establish asymptotic estimates on the second derivatives of $f_{\delta}$ which have the following form:

$$
\begin{aligned}
\left|\partial_{\mathbf{n}, \mathbf{n}} f_{\delta}(z)-\frac{1}{\delta^{2}}[f](x) \Phi^{\prime}(u)\right| & \leq \frac{C}{\delta} \\
\left|\partial_{\mathbf{n}, \mathbf{t}} f_{\delta}(z)\right| & \leq \frac{C}{\delta} \\
\left|\partial_{\mathbf{t}, \mathbf{t}} f_{\delta}(z)+\frac{1}{\delta}[f](x) \kappa(x) \Phi(u)\right| & \leq \frac{\omega(\delta)}{\delta}
\end{aligned}
$$

where $\lim _{\delta \rightarrow 0} \omega(\delta)=0$ and with the notation $z=U_{\delta}(x, u)$. The constant $C$ and the function $\omega$ depend only on $f$. The proof of these estimates is given in the appendix. As an immediate consequence, we obtain an asymptotic estimate of $K\left(d^{2} f_{\delta}\right)=\sqrt{\left|\operatorname{det}\left(d^{2} f_{\delta}\right)\right|}$ of the form

$$
\left|\delta^{\frac{3}{2}} K\left(d^{2} f_{\delta}(z)\right)-\sqrt{|\kappa(x)|}\right|[f](x)\left|\sqrt{\left|\Phi(u) \Phi^{\prime}(u)\right|}\right| \leq \omega(\delta),
$$

where $\lim _{\delta \rightarrow 0} \omega(\delta)=0$, and the function $\omega$ depends only on $f$. Using the notations

$$
g_{\delta}(z):=\delta^{\frac{3}{2}} K\left(d^{2} f_{\delta}(z)\right), \quad \lambda(x):=\sqrt{|\kappa(x)|}|[f](x)|, \quad \mu(u):=\sqrt{\left|\Phi(u) \Phi^{\prime}(u)\right|},
$$

we thus have

$$
\left|g_{\delta}(z)-\lambda(x) \mu(u)\right| \leq \omega(\delta),
$$

for all $x \in \Gamma_{\delta}^{0}, u \in[-1,1]$ and $\delta>0$ sufficiently small, with $z=U_{\delta}(x, u)$. We claim that for any continuous functions $\left(g_{\delta}, \lambda, \mu\right)$ satisfying (3.26), we have for any $\tau>0$,

$$
\lim _{\delta \rightarrow 0} \delta^{-1} \int_{\Gamma_{\delta}} g_{\delta}^{\tau}=\int_{\Gamma} \lambda(x)^{\tau} d x \int_{-1}^{1} \mu(u)^{\tau} d u,
$$

which is in our case equivalent to the estimate

$$
\lim _{\delta \rightarrow 0} \delta^{\frac{3}{2} \tau-1} \int_{\Gamma_{\delta}} K\left(d^{2} f_{\delta}\right)^{\tau}=\int_{\Gamma}|[f]|^{\tau}|\kappa|^{\tau / 2} \int_{\mathbb{R}}\left|\Phi \Phi^{\prime}\right|^{\tau / 2} .
$$

In order to prove (3.27), we may assume without loss of generality that $\tau=1$ up to replacing $\left(g_{\delta}, \lambda, \mu\right)$ by $\left(g_{\delta}^{\tau}, \lambda^{\tau}, \mu^{\tau}\right)$. We first express the jacobian matrix of $U_{\delta}$ using the bases $B_{1}=((\mathbf{t}(x), 0),(0,1))$ and $B_{2}=(\mathbf{t}(x), \mathbf{n}(x))$ for the tangent spaces of $\Gamma \times[-1,1]$ and $\Omega$. This gives the expression

$$
\left[d U_{\delta}(x, u)\right]_{B_{1}, B_{2}}=\left(\begin{array}{c|c}
1-\delta u \kappa(x) & 0 \\
\hline 0 & \delta
\end{array}\right)
$$


and therefore $\left|\operatorname{det}\left(d U_{\delta}(x, u)\right)\right|=\delta-\delta^{2} u \kappa(x)$. Since $B_{1}$ and $B_{2}$ are orthonormal bases, this quantity is the jabobian of $U_{\delta}$ at $(x, u)$, and therefore

$$
\int_{\Gamma_{\delta}} g_{\delta}=\delta \int_{\Gamma_{\delta}^{0} \times[-1,1]} g_{\delta}(x+\delta u \mathbf{n}(x))(1-\delta u \kappa(x)) d x d u
$$

Combining with (3.26), and using dominated convergence we obtain (3.27).

Step 5: summation of the different contributions. Summing up the contributions of $\Omega_{\delta}, \mathcal{P}_{\delta}$ and $\Gamma_{\delta}$, we reach the estimate

$$
\begin{aligned}
\int_{\Omega} K\left(d^{2} f_{\delta}\right)^{\tau} & =\int_{\Omega_{\delta}} K\left(d^{2} f_{\delta}\right)^{\tau}+\int_{\mathcal{P}_{\delta}} K\left(d^{2} f_{\delta}\right)^{\tau}+\int_{\Gamma_{\delta}} K\left(d^{2} f_{\delta}\right)^{\tau} \\
& =\left(\int_{\Omega \backslash \Gamma} K\left(d^{2} f\right)^{\tau}+\varepsilon_{1}(\delta)\right)+B(\delta) \delta^{2-2 \tau}+\delta^{1-\frac{3}{2} \tau}\left(\int_{\Gamma}|[f]|^{\tau}|\kappa|^{\tau / 2} \int_{\mathbb{R}}\left|\Phi \Phi^{\prime}\right|^{\tau / 2}+\varepsilon_{2}(\delta)\right), \\
& =\left(S_{p}(f)^{\tau}+\varepsilon_{1}(\delta)\right)+B(\delta) \delta^{2-2 \tau}+\delta^{1-\frac{3}{2} \tau}\left(E_{p}(f)^{\tau} C_{p, \phi}^{\tau}+\varepsilon_{2}(\delta)\right),
\end{aligned}
$$

where $\lim _{\delta \rightarrow 0} \varepsilon_{1}(\delta)=\lim _{\delta \rightarrow 0} \varepsilon_{2}(\delta)=0$ and $B(\delta)$ is uniformly bounded. This concludes the proof of Theorem 3.2 ,

\section{Relation with other works}

Theorem 3.2 allows us to extend the definition of $A_{2}(f)$ when $f$ is a cartoon function, according to

$$
A_{2}(f):=\left(S_{2}(f)^{2 / 3}+E_{2}(f)^{2 / 3} C_{2, \varphi}^{2 / 3}\right)^{3 / 2} .
$$

We first compare this additive form with the total variation $\operatorname{TV}(f)$. If $f$ is a cartoon function, its total variation has the additive form

$$
\operatorname{TV}(f):=\|\nabla f\|_{L^{1}(\Omega \backslash \Gamma)}+\|[f]\|_{L^{1}(\Gamma)},
$$

Both (4.29) and (4.30) include a "smooth term" and an "edge term". It is interesting to compare the edge term of $A_{2}(f)$, which is given by

$$
E_{2}(f)=\|\sqrt{|\kappa|}[f]\|_{L^{2 / 3}(\Gamma)},
$$

up to the multiplicative constant $C_{2, \varphi}$, with the one of $\operatorname{TV}(f)$ which is simply the integral of the jump

$$
J(f):=\|[f]\|_{L^{1}(\Gamma)},
$$

Both terms are 1-homogeneous with the value of the jump of the function $f$. In particular, if the value of this jump is 1 (for example when $f$ is the characteristic function of a set of boundary $\Gamma$ ), we have

$$
E_{2}(f)=\left(\int_{\Gamma}|\kappa|^{1 / 3}\right)^{3 / 2},
$$

while $J(f)$ coincides with the length of $\Gamma$. In summary, $A_{2}(f)$ takes into account the smoothness of edges, through their curvature $\kappa$, while $\operatorname{TV}(f)$ only takes into account their length.

Let us now investigate more closely the measure of smoothness of edges which is incorporated in $A_{2}(f)$. According to (4.31), this smoothness is meant in the sense that the arc length parametrizations of the curves that constitute $\Gamma$ admit second order derivatives in $L^{\frac{1}{3}}$. In the following, we show that this particular measure of smoothness is naturally related to some known results in two different areas: adaptive approximation of curves and affine-invariant image processing. 
We first revisit the derivation of the heuristic estimate (3.15) for the error between a cartoon function and its linear interpolation on an optimally adapted triangulation. In this computation, the contribution of the "edgy triangles" was estimated by the area of the layer $\Omega_{N}^{e}$ according to

$$
\left\|f-I_{\mathcal{T}_{N}} f\right\|_{L^{p}\left(\Omega_{N}^{e}\right)} \leq\|f\|_{L^{\infty}}\left|\Omega_{N}^{e}\right|^{1 / p} .
$$

Then we invoke the fact that $\Gamma$ is a finite union of $C^{2}$ curves $\Gamma_{j}$ in order to build a layer of global area $\left|\Omega_{N}^{e}\right| \leq C N^{-2}$, which results in the case $p=2$ into a contribution to the $L^{p}$ error of the order $\mathcal{O}\left(N^{-1}\right)$. The area of the layer $\Omega_{N}^{e}$ is indeed of the same order as the area between the edge $\Gamma$ and its approximation by a polygonal line with $\mathcal{O}(N)$ segments.

Each of the curves $\Gamma_{j}$ can be identified to the graph of a $C^{2}$ function in a suitable orthogonal coordinate system. If $\gamma$ is one of these functions, the area between $\Gamma$ and its polygonal approximation can thus be locally measured by the $L^{1}$ error between the one-dimensional function $\gamma$ and a piecewise linear approximation of this function. Since $\gamma$ is $C^{2}$, it is obvious that it can be approximated by a piecewise linear function on $\mathcal{O}(N)$ intervals with accuracy $\mathcal{O}\left(N^{-2}\right)$ in the $L^{\infty}$ norm and therefore in the $L^{1}$ norm. However, we may ask whether such a rate could be achieved under weaker conditions on the smoothness of $\gamma$. The answer to this question is a chapter of nonlinear approximation theory which identifies the exact conditions for a function $\gamma$ to be approximated at a certain rate by piecewise polynomial functions on adaptive one-dimensional partitions. We refer to [9 for a detailed treatment and only state the result which is of interest to us. We say that a function $\gamma$ defined on a bounded interval $I$ belongs to the approximation space $\mathcal{A}^{s}\left(L^{p}\right)$ if and only if there exists a sequence $\left(p_{N}\right)_{N>0}$ of functions where each $p_{N}$ is piecewise affine on a partition of $I$ by $N$ intervals such that

$$
\left\|\gamma-p_{N}\right\|_{L^{p}} \leq C N^{-s}
$$

For $0<s \leq 2$, it is known that $\gamma \in \mathcal{A}^{s}\left(L^{p}\right)$ provided that $\gamma \in B_{\tau, \tau}^{s}(I)$ with $\frac{1}{\tau}:=\frac{1}{p}+s$, where $B_{\tau, \tau}^{s}(I)$ is the standard Besov space that roughly describes those functions having $s$ derivatives in $L^{\tau}$. In the case $s=2$ and $p=1$ which is of interest to us, we find $\tau=\frac{1}{3}$ and therefore $\gamma$ should belong to the Besov space $B_{\frac{1}{3}, \frac{1}{3}}^{2}(I)$. Note that in our definition of cartoon functions, we assume much more than $B_{\frac{1}{3}, \frac{1}{3}}^{2}$ smoothness on $\gamma$, and it is not clear to us if Theorem (3.2) can be derived under this minimal smoothness assumption. However it is striking to see that the quantity $E_{2}(f)$ that is revealed by Theorem (3.2) precisely measures the second derivative of the arc-length parametrization of $\Gamma$ in the $L^{\frac{1}{3}}$ norm, up to the multiplicative weight $|[f]|^{2 / 3}$. Let us also mention that Besov spaces have been used in [10] in order to describe the smoothness of functions through the regularity of their level sets. Note that edges and level sets are two distinct concepts, which coincide in the case of piecewise constant cartoon functions.

The quantity $|\kappa|^{1 / 3}$ is also encountered in mathematical image processing, for the design of simple smoothing semi-groups that respect affine invariance with respect to the image. Since these semi-groups should also have the property of contrast invariance, they can be defined through curve evolution operators acting on the level sets of the image. The simplest curve evolution operator that respects affine invariance is given by the equation

$$
\frac{d \Gamma}{d t}=-|\kappa|^{1 / 3} \mathrm{n}
$$

where $\mathrm{n}$ is the outer normal, see e.g. 4. Here the value $1 / 3$ of the exponent plays a critical role. The fact that we also find it in $E_{2}(f)$ suggests that some affine invariance property also holds for this quantity as well as for $A_{2}(f)$. We first notice that if $f$ is a compactly supported $C^{2}$ function of two variables and $T$ is a bijective affine transformation, then with $\tilde{f}$ such that

$$
f=\tilde{f} \circ T
$$

we have the property

$$
d^{2} f(z)=L^{\mathrm{T}} d^{2} \tilde{f}(T z) L,
$$

where $L$ is the linear part of $T$ and $L^{\mathrm{T}}$ its transpose, so that

$$
\sqrt{\left|\operatorname{det}\left(d^{2} f(z)\right)\right|}=|\operatorname{det} L| \sqrt{\left|\operatorname{det}\left(d^{2} \tilde{f}(T z)\right)\right|} \text {. }
$$


By change of variable, we thus find that

$$
A_{p}(\tilde{f})=|\operatorname{det} L|^{1 / \tau-1} A_{p}(f)=|\operatorname{det} L|^{1 / p} A_{p}(f) .
$$

A similar invariance property can be derived on the interpolation error $\sigma_{N}(f)_{p}=\left\|f-I_{\mathcal{T}_{N}} f\right\|_{L^{p}}$ where $\mathcal{T}_{N}$ is a triangulation which is optimally adapted to $f$ in the sense of minimizing the linear interpolation error in the $L^{p}$ norm among all triangulations of cardinality $N$. We indeed remark that an optimal triangulation for $\tilde{f}$ is then given by applying $T$ to all elements of $\mathcal{T}_{N}$. For such a triangulation $\tilde{\mathcal{T}}_{N}:=T\left(\mathcal{T}_{N}\right)$, one has the commutation formula

$$
I_{\mathcal{T}_{N}} f=\left(I_{\tilde{\mathcal{T}}_{N}} \tilde{f}\right) \circ T
$$

and therefore we obtain by a change of variable that

$$
\sigma_{N}(\tilde{f})_{p}=\left\|\tilde{f}-I_{\tilde{\mathcal{T}}_{N}} \tilde{f}\right\|_{L^{p}}=|\operatorname{det} L|^{1 / p}\left\|f-I_{\mathcal{T}_{N}} f\right\|_{L^{p}}=|\operatorname{det} L|^{1 / p} \sigma_{N}(f)_{p} .
$$

Let us finally show that if $f$ is a cartoon function, then $E_{2}(f)$ satisfies a similar invariance property corresponding to $p=2$, namely

$$
E_{2}(\tilde{f})=|\operatorname{det} L|^{1 / 2} E_{2}(f) .
$$

Note that this cannot be derived by arguing that $A_{2}(f)$ satisfies this invariance property when $f$ and $\tilde{f}$ are smooth, since we lose the affine invariance property as we introduce the convolution by $\varphi_{\delta}$ : we do not have

$$
(f \circ T) * \varphi_{\delta}=\left(f * \varphi_{\delta}\right) \circ T .
$$

unless $T$ is a rotation or a translation.

Let $\Gamma_{j}$ be one of the $C^{2}$ pieces of $\Gamma$ and $\gamma_{j}:\left[0, B_{i}\right] \rightarrow \Omega$ a regular parametrisation of $\Gamma_{j}$. The curvature of $\Gamma$ on $\Gamma_{j}$ at the point $\gamma_{j}(t)$ is therefore given by

$$
\kappa\left(\gamma_{j}(t)\right)=\frac{\operatorname{det}\left(\gamma_{j}^{\prime}(t), \gamma_{j}^{\prime \prime}(t)\right)}{\left\|\gamma_{j}^{\prime}(t)\right\|^{3}}
$$

Since $f=\tilde{f} \circ T$, the discontinuity curves of $\tilde{f}$ are the images of those of $f$ by $T$ :

$$
\tilde{\Gamma}_{j}=T\left(\Gamma_{j}\right) .
$$

The curvature of $\tilde{\Gamma}_{j}$ at the point $T\left(\gamma_{j}(t)\right)$ is therefore given by

$$
\tilde{\kappa}\left(T\left(\gamma_{j}(t)\right)\right)=\frac{\operatorname{det}\left(L \gamma_{j}^{\prime}(t), L \gamma_{j}^{\prime \prime}(t)\right)}{\left\|L \gamma_{j}^{\prime}(t)\right\|^{3}}=\operatorname{det}(L) \frac{\operatorname{det}\left(\gamma_{j}^{\prime}(t), \gamma_{j}^{\prime \prime}(t)\right)}{\left\|L \gamma_{j}^{\prime}(t)\right\|^{3}}
$$

This leads us to the relation :

$$
|\operatorname{det}(L)|^{1 / 3}\left|\kappa\left(\gamma_{j}(t)\right)\right|^{1 / 3}\left\|\gamma_{j}^{\prime}(t)\right\|=\left|\tilde{\kappa}\left(T\left(\gamma_{j}(t)\right)\right)\right|^{1 / 3}\left\|L \gamma_{j}^{\prime}(t)\right\|,
$$

and therefore

$$
\begin{aligned}
\int_{\tilde{\Gamma}_{j}}|[\tilde{f}]|^{2 / 3}|\tilde{\kappa}|^{1 / 3} & =\int_{0}^{B_{j}}\left|[\tilde{f}]\left(T\left(\gamma_{j}(t)\right)\right)\right|^{2 / 3}\left|\tilde{\kappa}\left(T\left(\gamma_{j}(t)\right)\right)\right|^{1 / 3}\left\|L \gamma_{j}^{\prime}(t)\right\| d t \\
& =|\operatorname{det} L|^{1 / 3} \int_{0}^{B_{j}}\left|[f]\left(\gamma_{j}(t)\right)\right|^{2 / 3}\left|\kappa\left(\gamma_{j}(t)\right)\right|^{1 / 3}\left\|\gamma_{j}^{\prime}(t)\right\| d t \\
& =|\operatorname{det} L|^{1 / 3} \int_{\Gamma_{i}}|[f]|^{2 / 3}|\kappa|^{1 / 3}
\end{aligned}
$$

Summing over all $j=1, \cdots, l$ and elevating to the $3 / 2$ power we obtain (4.34). 

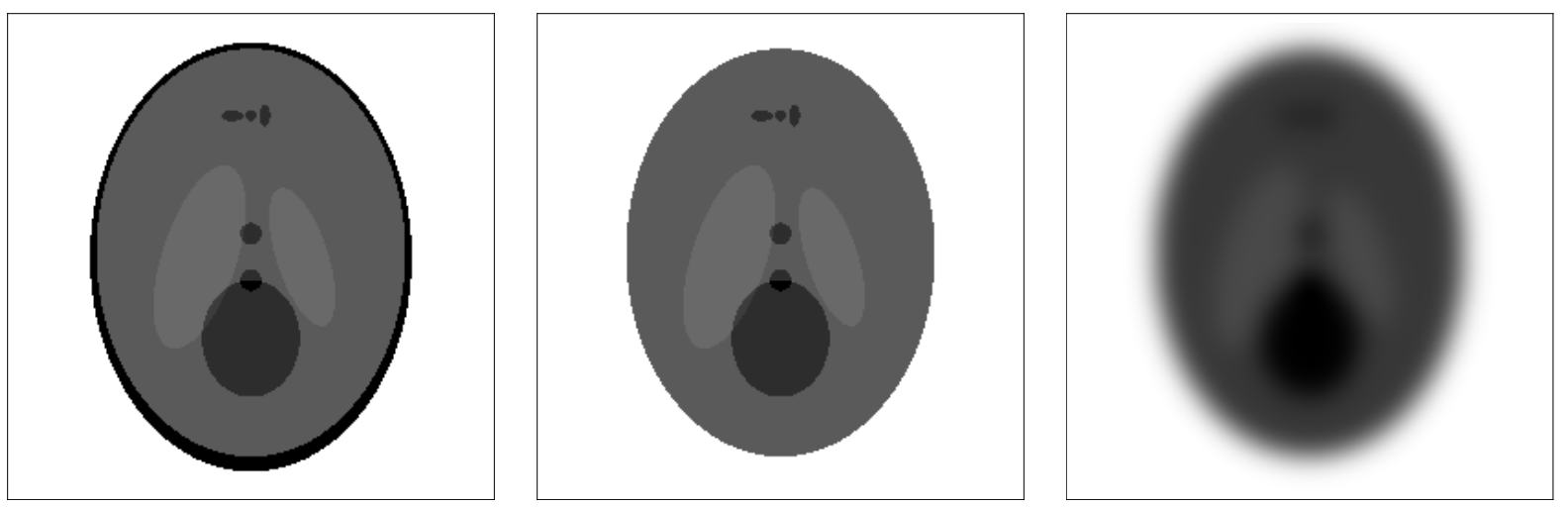

Figure 1: The Logan-Shepp image (left), modified image (center), smoothed image (right).

\section{$5 \quad$ Numerical tests}

In this section, we validate our previous results by numerical tests applied to a simple cartoon image: the Logan-Shepp phantom. We use a $256 \times 256$ pixel version of this image, with a slight modification which is motivated further. This image is iteratively smoothed by the numerical scheme

$$
u_{i, j}^{n+1}=\frac{u_{i, j}^{n}}{2}+\frac{u_{i+1, j}^{n}+u_{i-1, j}^{n}+u_{i, j+1}^{n}+u_{i, j-1}^{n}}{8}
$$

This scheme is an explicit discretization of the heat equation. Formally, as $n$ grows, $u^{n}$ is a discretization of

$$
u * \varphi_{\lambda \sqrt{n}} \text { with } \varphi_{\delta}(z):=\frac{1}{\pi \delta^{2}} e^{-\frac{\|z\|^{2}}{\delta^{2}}},
$$

where $u$ stands for the continuous image. The determinant of the hessian is discretised by the following 9 -points formula

$$
d_{i, j}^{n}:=\left(u_{i, j+1}^{n}-2 u_{i, j}^{n}+u_{i, j-1}^{n}\right)\left(u_{i+1, j}^{n}-2 u_{i, j}^{n}+u_{i-1, j}^{n}\right)-\frac{\left(u_{i+1, j+1}^{n}+u_{i-1, j-1}^{n}-u_{i+1, j-1}^{n}-u_{i-1, j+1}^{n}\right)^{2}}{16}
$$

For each value of $n$, we then compute the $\ell^{\tau}$ norm of the array $\left(\sqrt{\left|d_{i, j}^{n}\right|}\right)$ for $\tau \in\left[\frac{1}{2}, 1\right]$, which corresponds to $p \in[1, \infty]$ with $\frac{1}{\tau}:=1+\frac{1}{p}$. This norm is thus a discretization of the quantity

$$
\left\|\sqrt{\left|\operatorname{det}\left(d^{2}\left(u * \varphi_{\lambda \sqrt{n}}\right)\right)\right|}\right\|_{L^{\tau}} .
$$

For each value of $n$ we obtain a function $\tau \in\left[\frac{1}{2}, 1\right] \rightarrow D_{n}(\tau) \in \mathbb{R}_{+}$.

As $n$ grows, three consecutive but potentially overlapping phases appear in the behaviour of the functions $D_{n}$, which are illustrated on Figure 2 .

1. For small $n$, the 9-points discretisation is not a good approximation of the determinant of the hessian due to the fact that the pixel discretization is too coarse compared to the smoothing width. During this phase, the functions $D_{n}$ decay rapidly for all values of $\tau$.

2. For some range of $n$, the edges have been smoothed by the action of (5.37), but the parameter $\lambda \sqrt{n}$ in (5.38) remains rather small. Our previous analysis applies and we observe that $D_{n}(2 / 3)$ is (approximately) constant while $D_{n}(\tau)$ increases for $\tau<2 / 3$ and decreases for $\tau>2 / 3$.

3. For large $n$, the details of the picture fade and begin to disappear. The picture begins to resemble a constant picture. Therefore the functions $D_{n}$ decay for all values of $\tau$, and eventually tend to 0 . 

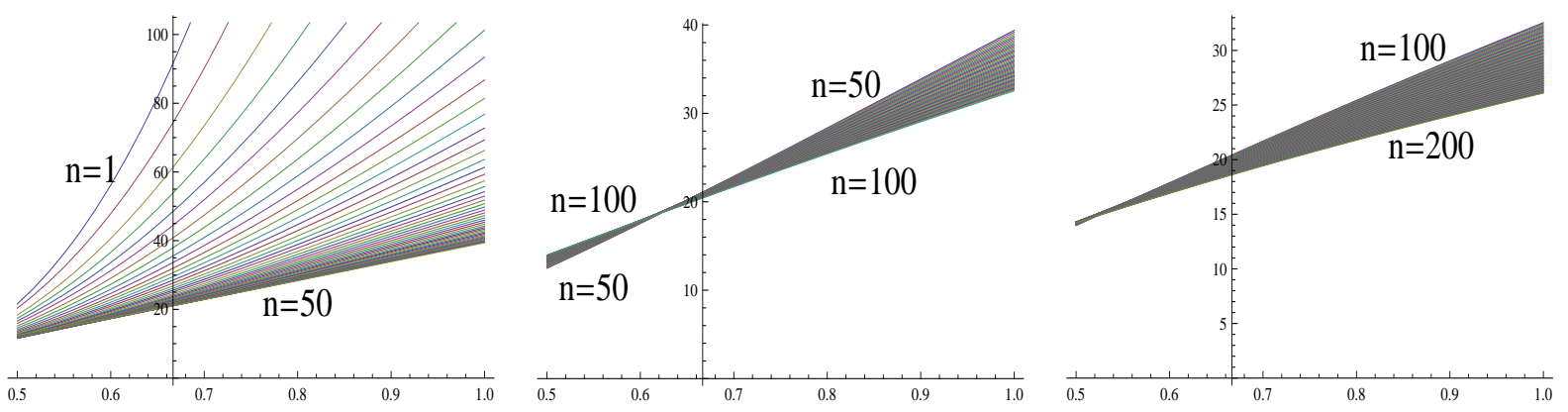

Figure 2: The curves $D_{n}(\tau)$ for $n \leq 50$ (left), $50 \leq n \leq 100$ (center) and $100 \leq n \leq 200$ (right).

In our numerical experiments, we used the well known Shepp-Logan Phantom with a slight modification as shown on Figure 1: we have removed the thin layer around the head, which represents the skull, because it disappears too quickly by the smoothing procedure and causes phases 1 and 3 to overlap, masking phase 2. For more complicated functions $f$, such as most photographic pictures, phases 1 and 3 also tend to overlap for similar reasons. Indeed, these pictures often have details at the pixel scale, including electronic noise due to the captor. Since these details disappear early phase 3 begins immediately, therefore phase 2 cannot be observed.

\section{Extension to higher dimensions and higher order elements}

The results on approximation by anisotropic bidimensional piecewise linear finite elements that we have exposed in $\S 2$ have been generalized in [19] to the case of elements of arbitrary order $m-1$ defined on partitions of $\Omega \subset \mathbb{R}^{\mathrm{d}}$ by simplices. Here, the local error is defined as

$$
e_{m, T}(f):=\left\|f-I_{m, T} f\right\|_{L^{P}(T)}
$$

where $I_{m, T}$ denotes the local interpolation operator on $\mathbb{P}_{m-1}$ for a $d$-dimensional simplex $T$. This operator is defined by the condition

$$
I_{m, T} v(\gamma)=v(\gamma)
$$

for all points $\gamma \in T$ with barycentric coordinates in the set $\left\{0, \frac{1}{m-1}, \frac{2}{m-1}, \cdots, 1\right\}$. Then one defines for any homogeneous polynomial $\mathbf{q} \in \mathbb{P}_{m}$,

$$
K_{m, p}(\mathbf{q}):=\inf _{|T|=1} e_{m, T}(\mathbf{q})_{p}
$$

We refer to $K_{m, p}$ as the shape function. For piecewise linear elements in dimension two, i.e. $m=d=2$, we have observed that $K_{p}=K_{2, p}$ has the special form given by (2.10) which justifies the introduction of the quantity $A_{p}(f)$. In a similar way, it can easily be proved that for piecewise linear elements in higher dimension, i.e. $m=2$ and $d>2$, one has

$$
c_{1}|\operatorname{det}(\mathbf{q})|^{1 / d} \leq K_{2, p}(\mathbf{q}) \leq c_{2}|\operatorname{det}(\mathbf{q})|^{1 / d} .
$$

For piecewise quadratic elements in dimension two, i.e. $m=3$ and $d=2$, it was proved in [19] that

$$
c_{1}|\operatorname{disc}(\mathbf{q})|^{1 / 4} \leq K_{3, p}(\mathbf{q}) \leq c_{2}|\operatorname{disc}(\mathbf{q})|^{1 / 4}
$$

for any homogeneous polynomial $\mathbf{q} \in \mathbb{P}_{3}$, where

$$
\operatorname{disc}\left(a x^{3}+b x^{2} y+c x y^{2}+d y^{3}\right):=b^{2} c^{2}-4 a c^{3}-4 b^{3} d+18 a b c d-27 a^{2} d^{2} .
$$

For other values of $m$ and $d$, equivalent expressions of $K_{m, p}(\mathbf{q})$ in terms of polynomials in the coefficients of $\mathbf{q}$ are available but of less simple form. 
Defining the finite element interpolation error by an optimally adapted partition

$$
\sigma_{N}(f)_{p}:=\inf _{\#(\mathcal{T}) \leq N}\left\|f-I_{m, \mathcal{T}} f\right\|_{L^{p}}
$$

where $I_{m, \mathcal{T}}$ is the global interpolation operator for the simplicial partition $\mathcal{T}$, the following generalization of (2.13) is proved in [19]:

$$
\limsup _{N \rightarrow+\infty} N^{\frac{m}{d}} \sigma_{N}(f)_{p} \leq C_{d}\left\|K_{m, p}\left(\frac{d^{m} f}{m !}\right)\right\|_{L^{\tau}(\Omega)}, \frac{1}{\tau}:=\frac{1}{p}+\frac{m}{d} .
$$

The constant $C_{d}$ is equal to 1 when $d=2$ but larger than 1 when $d>2$ due to the impossibility of exactly tiling the space with locally optimized simplices. If $f$ is a $C^{m}$ function of $d$ variables, it is therefore natural to consider the quantity

$$
A_{m, p}(f):=\left\|K_{m, p}\left(d^{m} f\right)\right\|_{L^{\tau}(\Omega)}, \frac{1}{\tau}:=\frac{1}{p}+\frac{m}{d},
$$

as a possible way to measuring anisotropic smoothness. For $d=2$ and piecewise linear elements, we have seen in $\S 2$ that $A_{2, p}(f)$ is equivalent to the quantity $A_{p}(f)$.

Similarly to $A_{p}$ we are interested in the possible extension of $A_{m, p}$ to cartoon functions. We first introduce a generalisation of the notion of cartoon functions to higher piecewise smoothness $m$ and dimension $d$.

Definition 6.1 Let $m \geq 2$ and $d \geq 2$ be two integers. Let $\Omega \subset \mathbb{R}^{d}$ be an open set. We say that a function $f$ defined on $\Omega$ is a $C^{m}$ cartoon function if it is almost everywhere of the form

$$
f=\sum_{1 \leq i \leq k} f_{i} \chi_{\Omega_{i}}
$$

where the $\Omega_{i}$ are disjoint open sets with piecewise $C^{2}$ boundary, no cusps (i.e. satisfying an interior and exterior cone condition), and such that $\bar{\Omega}=\cup_{i=1}^{k} \bar{\Omega}_{i}$. Additionally, for each $1 \leq i \leq k$, the function $f_{i}$ is assumed to be $C^{m}$ on $\bar{\Omega}_{i}$.

Let us consider a fixed cartoon function $f$ on a polyhedral domain $\Omega \subset \mathbb{R}^{d}$ (i.e. $\Omega$ is such that $\bar{\Omega}$ is a closed polyhedron), and a decomposition $\left(\Omega_{i}\right)_{1 \leq i \leq k}$ of $\Omega$ as in definition 6.1. As before we define $\Gamma:=\bigcup_{1 \leq i \leq k} \partial \Omega_{i}$, the union of the boundaries of the $\Omega_{i}$. Our assumptions on the sets $\left(\Omega_{i}\right)_{1 \leq i \leq k}$ imply that $\Gamma$ is the union of a finite number of open hypersurfaces $\left(\Gamma_{j}\right)_{1 \leq j \leq l}$, and of a set $\mathcal{P}$ of dimension $d-2$.

As in $\S 3$, we now consider a sequence $f_{N}$ of piecewise linear approximations of $f$ on simplicial partitions $\mathcal{T}_{N}$ of cardinality $N$. We distinguish two types of elements of $\mathcal{T}_{N}$. A simplex $T \in \mathcal{T}_{N}$ is called "regular" if $T \cap \Gamma=\emptyset$, and we denote the set of these simplices by $\mathcal{T}_{N}^{r}$. Other simplices are called "edgy" and their set is denoted by $\mathcal{T}_{N}^{e}$. We can again split $\Omega$ according to

$$
\Omega:=\left(\cup_{T \in \mathcal{T}_{N}^{r}} T\right) \cup\left(\cup_{T \in \mathcal{T}_{N}^{e}} T\right)=\Omega_{N}^{r} \cup \Omega_{N}^{e}
$$

Heuristically, if the partitions $\mathcal{T}_{N}$ are built with approximation error minimisation in mind, the number of elements should be balanced between $\mathcal{T}_{N}^{r}$ and $\mathcal{T}_{N}^{e}$. The partition $\mathcal{T}_{N}^{r}$ tends to cover most of the surface of $\Omega$, with simplices of diameter $\leq C N^{-\frac{1}{d}}$, and $L^{\infty}$ approximation error $\left|f-f_{N}\right| \leq C N^{-\frac{m}{d}}$ (since we use $\mathcal{P}_{m-1}$ elements). On the other hand, since $f$ has discontinuities along $\Gamma$, the $L^{\infty}$ approximation error on $\mathcal{T}_{N}^{e}$ does not tend to zero, and $\mathcal{T}_{N}^{e}$ should thus be chosen so as to produce a thin layer around $\Gamma$. Let $h$ be the typical diameter of an element of $\mathcal{T}_{N}^{e}$. Since the $\Gamma_{j}$ has bounded curvature, this layer can be made of width $\mathcal{O}\left(h^{2}\right)$ and therefore the layer around $\Gamma$ has volume bounded by $h^{2} \mathcal{H}_{d-1}(\Gamma)$ up to a fixed multiplicative constant, where $\mathcal{H}_{d-1}(\Gamma)$ is the $d-1$ dimensional Hausdorff measure of $\Gamma$. On the other hand the minimal number of such elements of diameters $h$ needed to cover $\Gamma$ is bounded by $h^{1-d} \mathcal{H}_{d-1}(\Gamma)$ up to a fixed multiplicative constant. Eventually, we find that the layer around $\Gamma$ has volume bounded by $C N^{-\frac{2}{d-1}}$.

Hence we have the following heuristic error estimate, for a well designed anisotropic partition:

$$
\begin{aligned}
\left\|f-f_{N}\right\|_{L^{p}(\Omega)} & \leq\left\|f-f_{N}\right\|_{L^{p}\left(\Omega_{N}^{r}\right)}+\left\|f-f_{N}\right\|_{L^{p}\left(\Omega_{N}^{e}\right)} \\
& \leq\left\|f-f_{N}\right\|_{L^{\infty}\left(\Omega_{N}^{r}\right)}\left|\Omega_{N}^{r}\right|^{\frac{1}{p}}+\left\|f-f_{N}\right\|_{L^{\infty}\left(\Omega_{N}^{e}\right)}\left|\Omega_{N}^{e}\right|^{\frac{1}{p}} \\
& \leq C\left(N^{-\frac{m}{d}}+N^{\frac{-2}{p(d-1)}}\right)
\end{aligned}
$$


This leads us to define a critical exponent

$$
p_{c}=p_{c}(m, d):=\frac{2 d}{m(d-1)} .
$$

If one measures the error in $L^{p}$ norm with $p>p_{c}(m, d)$, then the contribution of the edge neighbourhood $\Omega_{N}^{e}$ dominates, while if $p<p_{c}(m, d)$ it is negligible compared to the contribution of the smooth region $\Omega_{N}^{r}$. For the critical exponent $p=p_{c}(m, d)$ the two terms have the same order, which makes the situation more interesting. Note in particular that $p_{c}(2,2)=2$, which is consistent with our previous analysis.

For $p \leq p_{c}(m, d)$, we obtain the approximation rate $N^{-m / d}$ which suggests that approximation results such as (6.39) should also apply to cartoon functions and that the quantity $A_{m, p}(f)$ should be finite for such functions. We again need to use a regularization approach, for the same reasons as in $\S 3$. For a given dimension $d$, we consider a radial nonnegative function $\varphi$ of unit integral and supported in the unit ball of $\mathbb{R}^{d}$, and define for $\delta>0$

$$
\varphi_{\delta}(z):=\frac{1}{\delta^{d}} \varphi\left(\frac{z}{\delta}\right) \text { and } f_{\delta}=f * \varphi_{\delta} .
$$

In order to define the quantities of involved in our conjecture, we need to introduce the second fundamental form of a hypersurface. At any point $x \in \Gamma \backslash \mathcal{P}$ be denote by $\mathbf{n}(x)$ the unit normal to $\Gamma$. Note that since $\Gamma$ is piecewise $C^{2}$, the map $x \mapsto \mathbf{n}(x)$ is $C^{1}$ on $\Gamma \backslash \mathcal{P}$. Furthermore, we define $T_{x} \Gamma:=\mathbf{n}(x)^{\perp}$, the tangent space to $\Gamma$ at $x$. In a neighbourhood of $x \in \Gamma \backslash \mathcal{P}$, the hypersurface $\Gamma$ admits a parametrization of the form

$$
u \in T_{x} \Gamma \mapsto x+u+\lambda(u) \mathbf{n}(x) \in \Gamma \backslash \mathcal{P},
$$

where $\lambda$ is a scalar valued $C^{2}$ function. By definition, the second fundamental form of $\Gamma$ at the point $x$ is the quadratic form $\Pi_{x}$ associated to $d^{2} \lambda(0)$ which is defined on $T_{x} \Gamma \times T_{x} \Gamma$. Alternatively, for all $u, v \in T_{x} \Gamma$ we have $\Pi_{x}(u, v):=-\left\langle\partial_{u} \mathbf{n}, v\right\rangle$. The Gauss curvature $\kappa(x)$ is the determinant of $\Pi_{x}$, in any orthonormal basis of $T_{x} \Gamma$,

$$
\kappa(z):=\operatorname{det} \Pi_{z} .
$$

For example, in two space dimensions the tangent space $T_{x} \Gamma$ is one dimensional, and we simply have $\Pi_{x}(u, v)=\kappa(x)\langle u, v\rangle$. We also denote by $\sigma(z) \in\{0, \cdots, d-1\}$ the signature of the quadratic form $\Pi_{z}$, which is defined as the number of its positive eigenvalues.

With $\tau$ such that $\frac{1}{\tau}:=\frac{m}{d}+\frac{1}{p}$, we define

$$
S_{p}(f):=\left\|K\left(d^{m} f\right)\right\|_{L^{\tau}(\Omega \backslash \Gamma)}=A_{p}\left(f_{\mid \Omega \backslash \Gamma}\right) .
$$

We conjecture the following generalization to Theorem 3.2 .

Conjecture 6.2 There exists d positive constants $C(k), k \in\{0, \cdots, d-1\}$, that depend on $\varphi, p, m, d$, such that, with

$$
E_{p}(f):=\left\|C(\sigma)|\kappa|^{\frac{m}{2 d}}[f]\right\|_{L^{\tau}(\Gamma)}=\left(\left.\left.\int_{\Gamma}|C(\sigma(z))| \kappa(z)\right|^{\frac{m}{2 d}}[f(z)]\right|^{\tau} d z\right)^{\frac{1}{\tau}}
$$

we have

- If $p<p_{c}$ then

$$
\lim _{\delta \rightarrow 0} A_{m, p}\left(f_{\delta}\right)=S_{p}(f)
$$

- If $p=p_{c}$ then

$$
\lim _{\delta \rightarrow 0}\left(A_{m, p}\left(f_{\delta}\right)\right)=\left(S_{p}(f)^{\tau}+E_{p}(f)^{\tau}\right)^{1 / \tau} .
$$

- If $p>p_{c}$ then

$$
\lim _{\delta \rightarrow 0} \delta^{\frac{1}{p_{c}}-\frac{1}{p}} A_{m, p}\left(f_{\delta}\right)=E_{p}(f) .
$$


In the remainder of this section, we give some arguments that justify this conjecture. Given a cartoon function $f$, we define the sets $\Omega_{\delta}, \Gamma_{\delta}^{0}, \Gamma_{\delta}$ and $\mathcal{P}_{\delta}$ similarly to $\S 3$. We need to perform an asymptotic analysis of the integral

$$
\int_{\Omega} K\left(d^{m} f_{\delta}\right)^{\tau}=\int_{\Omega_{\delta}} K\left(d^{m} f_{\delta}\right)^{\tau}+\int_{\mathcal{P}_{\delta}} K\left(d^{m} f_{\delta}\right)^{\tau}+\int_{\Gamma_{\delta}} K\left(d^{m} f_{\delta}\right)^{\tau} .
$$

As in the proof of Theorem 3.2 the contribution of $\mathcal{P}_{\delta}$ can be proved to be negligible compared with those of $\Omega_{\delta}$ and $\Gamma_{\delta}$ as $\delta \rightarrow 0$. The contribution of $\Omega_{\delta}$ satisfies

$$
\lim _{\delta \rightarrow 0} \int_{\Omega_{\delta}} K\left(d^{m} f_{\delta}\right)^{\tau}=\int_{\Omega \backslash \Gamma} K\left(d^{m} f\right)^{\tau} .
$$

The main difficulty lies again in the contribution of $\Gamma_{\delta}$. Let us define $\tau_{c}$ by

$$
\frac{1}{\tau_{c}}:=\frac{m}{d}+\frac{1}{p_{c}}
$$

The contribution of $\Gamma_{\delta}$ can be computed if one can establish an estimate generalizing (3.25) according to

$$
\left.\left|\delta^{\frac{1}{\tau_{c}}} K_{m, p}\left(d^{m} f_{\delta}(z)\right)-\right|[f](x)|| \kappa(x)\right|^{\frac{m}{2 d}} \Phi_{m, d, \sigma(x)}(u) \mid \leq \omega(\delta)
$$

where $\omega(\delta) \rightarrow 0$ as $\delta \rightarrow 0, x \in \Gamma_{\delta}^{0}, u \in[-1,1], z=x+\delta u \mathbf{n}(x)$, and where the function $\Phi_{m, d, k}:[-1,1] \rightarrow \mathbb{R}$ only depends on $m, d, k$ and $\varphi$. If (6.43) holds, we then easily derive that

$$
\lim _{\delta \rightarrow 0} \delta^{\frac{\tau}{\tau_{c}}-1} \int_{\Gamma_{\delta}} K\left(d^{m} f_{\delta}\right)^{\tau}=\int_{\Gamma} C(\sigma)|\kappa|^{\frac{\tau m}{2 d}}|[f]|^{\tau},
$$

with $C(k):=\int_{-1}^{1}\left|\Phi_{m, d, k}(u)\right|^{\tau} d u$, which leads to the proof of the conjecture.

We do not have a general proof of (6.43) for any $m, p$ and $d$. In the following, we justify its validity in two particular cases for which the explicit expression of $K_{m, p}$ is known to us: piecewise quadratic in two space dimensions $(d=2$ and $m=3)$ and piecewise linear in any dimension $(m=2)$.

Piecewise quadratic elements in two dimensions. For all $\delta>0, x \in \Gamma_{\delta}^{0}$ and $u \in[-1,1]$, let $\pi_{x, \delta, u} \in \mathbb{P}_{3}$ be the homogeneous cubic polynomial on $\mathbb{R}^{2}$ corresponding to $d^{3} f_{\delta}(x+\delta u \mathbf{n}(x))$. Let also $\pi_{x, u} \in \mathbb{P}_{3}$ be the homogeneous cubic polynomial on $\mathbb{R}^{2}$ defined by

$$
\pi_{x, u}(\lambda \mathbf{n}(x)+\mu \mathbf{t}(x))=-\lambda\left(\Phi^{\prime \prime}(u) \lambda^{2}-3 \Phi^{\prime}(u) \kappa(x) \mu^{2}\right)
$$

for all $(\lambda, \mu) \in \mathbb{R}^{2}$, where $\Phi$ is defined by (3.17). For all $x \in \Gamma$, we denote by $M_{x, \delta}$ the (symmetric) linear map defined by

$$
M_{x, \delta} \mathbf{n}(x)=\delta \mathbf{n}(x) \text { and } M_{x, \delta} \mathbf{t}(x)=\sqrt{\delta} \mathbf{t}(x) .
$$

Then, using a reasoning similar to the one used in the appendix of this paper, it can be proved that

$$
\left\|\pi_{x, \delta, u} \circ M_{x, \delta}-[f](x) \pi_{x, u}\right\| \leq \omega(\delta) .
$$

where $\lim _{\delta \rightarrow 0} \omega(\delta)=0$ and the function $\omega$ depends only on $f$. Furthermore, it is proved in [19] that

$$
K_{3, p}(\mathbf{q})=C \sqrt[4]{|\operatorname{disc} \mathbf{q}|}
$$

where the positive constant $C$ depends on $p$ and the sign of disc q. Combining this expression with (6.44) proves the Estimate (6.43) and thus the conjecture in the case $m=3$ and $d=2$. 
Piecewise linear elements in any dimension. We use the second fundamental form of the discontinuity set $\Gamma$ in order to evaluate $d^{m} f_{\delta}$ on $\Gamma_{\delta}$. Characteristic functions are one of the simplest types of cartoon functions. In that case, it is possible to establish a simple relation between the second fundamental form of the edge set and the second derivatives of $f$ in a distributional sense: if $\Omega \subset \mathbb{R}^{d}$ is a bounded domain with smooth boundary $\Gamma$ and inward normal $\mathbf{n}$, we then have for all $C^{2}$ test function $\psi$ and $u, v \in \mathbb{R}^{d}$

$$
-\int_{\Omega} \partial_{u, v}^{2} \psi=\int_{\Gamma}\langle u, \mathbf{n}\rangle\langle v, \mathbf{n}\rangle\left(\partial_{\mathbf{n}} \psi-\operatorname{tr}(\Pi) \psi\right)+\Pi^{\prime}(u, v) \psi,
$$

where $\Pi_{x}^{\prime}(u, v)$ is the second fundamental form $\Pi_{x}$ applied to the orthogonal projection of $u$ and $v$ on $T_{x} \Gamma$. The proof of this formula (that generalizes (7.52) which is proved in the appendix and corresponds to the two-dimensional case $d=2$ ) is given further below. For all $x \in \Gamma$, we denote by $M_{x, \delta}$ the (symmetric) linear map defined by

$$
M_{x, \delta} \mathbf{n}(x)=\delta \mathbf{n}(x) \text { and } M_{x, \delta} \mathbf{t}=\sqrt{\delta} \mathbf{t}
$$

for all $\mathbf{t} \in T_{x} \Gamma$. For all $\delta>0, x \in \Gamma_{\delta}^{0}$ and $u \in[-1,1]$, let $\pi_{x, \delta, u} \in \mathbb{P}_{2}$ be the homogeneous quadratic polynomial on $\mathbb{R}^{d}$ corresponding to $d^{2} f_{\delta}(x+\delta u \mathbf{n}(x))$. Let also $\pi_{x, u} \in \mathbb{P}_{2}$ be the homogeneous quadratic polynomial on $\mathbb{R}^{d}$ defined by

$$
\pi_{x, u}(\lambda \mathbf{n}(x)+\mathbf{t})=\Phi^{\prime}(u) \lambda^{2}-\Phi(u) \Pi_{x}(\mathbf{t}, \mathbf{t})
$$

for all $\mathbf{t} \in T_{x} \Gamma$, where $\Phi(x):=\int_{\mathbb{R}^{d-1}} \varphi(x, y) d y$. Then, using formula (6.45) and a reasoning analogous to the one presented in the appendix, it can be proved that

$$
\left\|\pi_{x, \delta, u} \circ M_{x, \delta}-[f](x) \pi_{x, u}\right\| \leq \omega(\delta) .
$$

where $\lim _{\delta \rightarrow 0} \omega(\delta)=0$ and the function $\omega$ depends only on $f$. Furthermore, it is proved in [19] that

$$
K_{2, p}(\mathbf{q})=C \sqrt[d]{|\operatorname{det} \mathbf{q}|}
$$

where the positive constant $C$ depends on $d, p$ and the signature of $\mathbf{q}$. Combining this expression with (6.46) proves the estimate (6.43) and thus the conjecture in the case $m=2$ in any dimension $d>1$.

Proof of (6.45): Let $\mathrm{P}_{\Gamma}$ be the orthogonal projection onto $\Gamma$, and for all $x \in \Gamma$ let $\mathrm{P}_{x}$ be the orthogonal projection onto $T_{x} \Gamma$. We consider a vector $u \in \mathbb{R}^{d}$ and we define $\mathrm{U}: \Gamma \rightarrow \Gamma$ by $\mathrm{U}(x):=\mathrm{P}_{\Gamma}(x+u)$. If $\|u\|\|\Pi\|_{L^{\infty}(\Gamma)}<1$, then $\mathrm{U}$ is smooth and its differential $d_{x} \mathrm{U}: T_{x} \Gamma \rightarrow T_{x^{\prime}} \Gamma$, where

$$
x^{\prime}=\mathrm{U}(x),
$$

is given by the following formula

$$
d_{x} \mathrm{U}=\left(\mathrm{Id}-\left\langle u, \mathbf{n}\left(x^{\prime}\right)\right\rangle \Pi_{x^{\prime}}\right)^{-1} \mathrm{P}_{x^{\prime}} .
$$

The determinant of $d_{x} U$ (more precisely the determinant of the matrix of $d_{x} U$ in direct orthogonal bases of $T_{x} \Gamma$ and $\left.T_{x^{\prime}} \Gamma\right)$ is

$$
\operatorname{det}\left(d_{x} \mathrm{U}\right)=\operatorname{det}\left(\operatorname{Id}-\left\langle u, \mathbf{n}\left(x^{\prime}\right)\right\rangle \Pi_{x^{\prime}}\right)^{-1}\left\langle\mathbf{n}(x), \mathbf{n}\left(x^{\prime}\right)\right\rangle=1+\left\langle u, \mathbf{n}\left(x^{\prime}\right)\right\rangle \operatorname{tr}\left(\Pi_{x^{\prime}}\right)+\|u\| \omega_{1}(u, x) .
$$

where $\omega_{1}(u, x)$ tends uniformly to 0 as $u \rightarrow 0$. Furthermore, it is easy to show that

$$
\left|\psi(x+u)-\psi\left(x^{\prime}\right)-\left\langle u, \mathbf{n}\left(x^{\prime}\right)\right\rangle \partial_{\mathbf{n}\left(x^{\prime}\right)} \psi\left(x^{\prime}\right)\right| \leq C\|u\|^{2},
$$

and $\left\|n\left(x^{\prime}\right)-n(x)-\Pi_{x^{\prime}}\left(\mathrm{P}_{x^{\prime}}(u)\right)\right\| \leq\|u\| \omega_{2}(u)$, where $C$ and $\omega_{2}$ are independent of $x \in \Gamma$ and $\omega_{2}(u) \rightarrow 0$ as $u \rightarrow 0$. Combining these results, we obtain

$$
\begin{gathered}
\int_{\Gamma} \psi(x+u)\langle\mathbf{n}(x), v\rangle d x=\int_{\Gamma} \psi(x+u)\langle\mathbf{n}(x), v\rangle \operatorname{det}\left(d_{x^{\prime}} \mathrm{U}\right)^{-1} d x^{\prime} \\
=\int_{\Gamma}\left\langle\mathbf{n}\left(x^{\prime}\right), v\right\rangle \psi\left(x^{\prime}\right) d x^{\prime}+\int_{\Gamma}\left\langle\mathbf{n}\left(x^{\prime}\right), v\right\rangle\left\langle\mathbf{n}\left(x^{\prime}\right), u\right\rangle\left(\partial_{\mathbf{n}\left(x^{\prime}\right)} \psi-\operatorname{tr}\left(\Pi_{x}^{\prime}\right) \psi\left(x^{\prime}\right)\right)+\left\langle v, \Pi_{x}^{\prime}\left(\mathrm{P}_{x^{\prime}}(u)\right)\right\rangle \psi\left(x^{\prime}\right) d x^{\prime}+\|u\| \omega_{3}(u),
\end{gathered}
$$


where $\omega_{3}(u) \rightarrow 0$ as $u \rightarrow 0$. We conclude the proof of (6.45) using the formula

$$
-\int_{\Omega} \partial_{u, v}^{2} \psi=\lim _{h \rightarrow 0} h^{-1} \int_{\Gamma}(\psi(x+h u)-\psi(x))\langle\mathbf{n}(x), v\rangle d x .
$$

Remark 6.3 Similarly to the results presented in $\S 4$, there is an affine invariance property associated to $\kappa$ : if $T$ is an affine transformation of $\mathbb{R}^{d}$ with linear part $L$, and if $f=\tilde{f} \circ T, \tilde{\Gamma}=T(\Gamma)$ and $\tilde{\kappa}$ is the Gauss curvature of $\tilde{\Gamma}$, then one has for any $s \geq 0$,

$$
(\operatorname{det} L)^{\frac{d-1}{d+1}} \int_{\Gamma}|C(\sigma)[f]|^{s}|\kappa|^{\frac{1}{d+1}}=\int_{\tilde{\Gamma}}|C(\tilde{\sigma})[\tilde{f}]|^{s}|\tilde{\kappa}|^{\frac{1}{d+1}} .
$$

It follows from this observation that when $p=p_{c}$, the contribution of the edges is affine invariant in the sense that

$$
E_{p_{c}}(\tilde{f})=(\operatorname{det} L)^{\frac{d-1}{d+1}} E_{p_{c}}(f) .
$$

Since one also has $A_{m, p}(\tilde{f})=(\operatorname{det} L)^{\frac{d-1}{d+1}} A_{m, p}(f)$ this comforts the conjecture. Let us mention that the quantity $|\kappa|^{\frac{1}{d+1}}$ has been used in [21] in order to define surface smoothing operators that are invariant under affine change of coordinates.

\section{Conclusion}

In this paper we have investigated the quantity $A_{p}(f)$ which governs the rate of approximation by anisotropic $\mathbb{P}_{1}$ finite elements as a way to describe anisotropic smoothness of functions. This quantity is not a semi-norm due to the presence of the non-linear quantity $\operatorname{det}\left(d^{2} f\right)$ and cannot be defined in a straightforward manner for general distributions. We nevertheless have shown that this quantity can be defined for cartoon images with geometrically smooth edges when $p \leq 2$. A theoretical issue remains to give a satisfactory meaning to the full class of functions for which this quantity is finite.

From a more applied perspective, it could be interesting to investigate the role of $A_{p}(f)$ in problems where anisotropic features naturally arise:

1. Approximation of PDE's: in the case of one dimensional hyperbolic conservation laws, it was proved in [1] that despite the appearance of discontinuities the solution has high order smoothness in Besov spaces that govern the rate of adaptive approximation by piecewise polynomials. A natural question is to ask wether similar results hold in higher dimension, which corresponds to understanding if $A_{p}(f)$ remains bounded despite the appearance of shocks.

2. Image processing: as illustrated in $\S 5$, the quantity $A_{p}(f)$ can easily be discretized and defined for pixelized images. It is therefore tempting to use $A_{2}(f)$ in a similar way as the total variation in (1.2), by solving a problem of the form

$$
\min _{g \in B V}\left\{A_{2}(g) ;\|T g-h\|_{L^{2}} \leq \varepsilon\right\},
$$

with the objective of promoting images with piecewise smooth edges. The main difficulty is that $A_{2}$ is not a convex functional. One way to solve this difficulty could be to reformulate (7.47) in a Bayesian framework as the search of a maximum of an a-posteriori probability distribution (MAP) as an estimator of $f$. In this framework, we may instead search for a minimal mean-square error estimator (MMSE), and this search can be implemented by stochastic algorithms which does not require the convexity of $A_{2}$, see [18]. 


\section{Appendix: proof of the estimates (3.22) $-((3.23)-(3.24)$.}

It is known since the work of Whitney on extension theorems (see in particular 22]) that for any open set $U \subset \mathbb{R}^{d}$, and any $g \in C^{2}(\bar{U})$ there exists $\tilde{g} \in C^{2}\left(\mathbb{R}^{d}\right)$ such that $\tilde{g}_{\mid U}=g$. It follows that for each $1 \leq i \leq k$, there exists $\tilde{f}_{i} \in C^{2}\left(\mathbb{R}^{2}\right)$, compactly supported, and such that $\tilde{f}_{i \mid \Omega_{i}}=f_{i}$.

Let $\Gamma_{j}$ be one of the pieces of $\Gamma$, between the domains $\Omega_{k}$ and $\Omega_{l}$, and let $s=\tilde{f}_{k}$ and $t=\tilde{f}_{l}-\tilde{f}_{k}$. Although the domains $\Omega_{k}$ and $\Omega_{l}$ are only piecewise smooth, there exists an open set $\Omega^{\prime}$ with $C^{2}$ boundary such that for $\delta_{0}>0$ small enough

$$
f=s \chi_{\Omega^{\prime}}+t \text { on } \bigcup_{0<\delta \leq \delta_{0}}\left(\Gamma_{j, \delta}+B_{\delta}\right),
$$

where $B_{\delta}$ is the ball of radius $\delta$ centered at 0 . Note that $\Gamma_{j} \subset \Gamma^{\prime}:=\partial \Omega^{\prime}$ and that $s=[f]$ on $\Gamma_{j}$. In the following, the variables $x, z$ are always subject to the restriction

$$
x \in \Gamma_{j, \delta}^{0} \text { and } z=U_{\delta}(x, u)=x+\delta u \mathbf{n}(x) \text { where } 0<\delta \leq \delta_{0} \text { and }|u| \leq 1,
$$

note that $z \in \Gamma_{j, \delta}$ and $\|x-z\| \leq \delta$. We therefore have

$$
f_{\delta}(z)=\int_{\Omega^{\prime}} s(\tilde{x}) \varphi_{\delta}(z-\tilde{x}) d \tilde{x}+t_{\delta}(z)
$$

where $t_{\delta}:=t * \varphi_{\delta}$. The second derivatives of $t_{\delta}$ are uniformly bounded, and are therefore negligible in regard of all three estimates (3.22), (3.23) and (3.24), indeed

$$
\left\|d^{2} t_{\delta}\right\|_{L^{\infty}}=\left\|\left(d^{2} t\right) * \varphi_{\delta}\right\|_{L^{\infty}} \leq\left\|d^{2} t\right\|_{L^{\infty}}\left\|\varphi_{\delta}\right\|_{L^{1}}=\left\|d^{2} t\right\|_{L^{\infty}}\|\varphi\|_{L^{1}}<\infty .
$$

We now define the $2 \times 2$ symmetric matrices

$$
I(z, x):=\int_{\Omega^{\prime}}(s(\tilde{x})-s(x)) d^{2} \varphi_{\delta}(z-\tilde{x}) d \tilde{x} \text { and } J(z):=\int_{\Omega^{\prime}} d^{2} \varphi_{\delta}(z-\tilde{x}) d \tilde{x}
$$

so that

$$
d^{2} f_{\delta}(z)=d^{2} t_{\delta}+I(z, x)+[f](x) J(z) .
$$

We already know that the contribution of $d^{2} t_{\delta}$ is negligible. We now prove that the same holds for the contribution of $I(z, x)$. Since $\varphi_{\delta}(z-\tilde{x})$ is non-zero only if $\|\tilde{x}-z\| \leq \delta$ and therefore $\|\tilde{x}-x\| \leq 2 \delta$, we can bound the norm of the matrix $I(z, x)$ by

$$
\|I(z, x)\| \leq 2 \delta\|d s\|_{L^{\infty}}\left\|d^{2} \varphi_{\delta}\right\|_{L^{1}} \leq 2 \delta\|d s\|_{L^{\infty}}\left\|d^{2} \varphi\right\|_{L^{1}} \delta^{-2}=C \delta^{-1} .
$$

This proves that the contribution of $I(z, x)$ is negligible for the two estimates (3.22) and (3.23). In order to prove that it is also negligible in the estimate (3.24), we need a finer analysis of $\mathbf{t}(x)^{\mathrm{T}} I(z, x) \mathbf{t}(x)$. For this purpose we fix a unit vector $u$ and the pair $(x, z)$. We introduce

$$
\Lambda(\tilde{x}):=(s(\tilde{x})-s(x)) \partial_{u} \varphi_{\delta}(z-\tilde{x})+\partial_{u} s(\tilde{x}) \varphi_{\delta}(z-\tilde{x}),
$$

so that by Leibniz rule

$$
(s(\tilde{x})-s(x)) \partial_{u, u}^{2} \varphi_{\delta}(z-\tilde{x})=\partial_{u, u}^{2} s(\tilde{x}) \varphi_{\delta}(z-\tilde{x})-\partial_{u} \Lambda(\tilde{x}) .
$$

Therefore

$$
\begin{aligned}
u^{\mathrm{T}} I(z, x) u & =\int_{\Omega^{\prime}}\left(\partial_{u, u}^{2} s(\tilde{x}) \varphi_{\delta}(z-\tilde{x})-\partial_{u} \Lambda(\tilde{x})\right) d \tilde{x} \\
& =\int_{\Omega^{\prime}} \partial_{u, u}^{2} s(\tilde{x}) \varphi_{\delta}(z-\tilde{x}) d \tilde{x}-\int_{\Gamma^{\prime}} \Lambda(\tilde{x})\langle\mathbf{n}(\tilde{x}), u\rangle d \tilde{x}
\end{aligned}
$$


The first integral clearly satisfies

$$
\left|\int_{\Omega^{\prime}} \partial_{u, u}^{2} s(\tilde{x}) \varphi_{\delta}(z-\tilde{x}) d \tilde{x}\right| \leq\left\|d^{2} s\right\|_{L^{\infty}}\left\|\varphi_{\delta}\right\|_{L^{1}},
$$

and is therefore bounded independently of $\delta$. We estimate the second integral for the special case $u=\mathbf{t}(x)$, remarking that $|\langle\mathbf{n}(\tilde{x}), \mathbf{t}(x)\rangle| \leq C_{1} \delta$ on the domain of integration. Therefore

$$
\left|\int_{\Gamma^{\prime}} \Lambda(\tilde{x})\langle\mathbf{n}(\tilde{x}), \mathbf{t}(x)\rangle d \tilde{x}\right| \leq C_{1} \delta\left|\Gamma^{\prime} \cap B(z, \delta)\right|\|\Lambda\|_{L^{\infty}},
$$

where, slightly abusing notations, we denote by $\left|\Gamma^{\prime} \cap B(z, \delta)\right|$ the length (1-dimensional Hausdorff measure) of the curve $\Gamma^{\prime} \cap B(z, \delta)$. Clearly $\Lambda(\tilde{x})=0$ if $\|z-\tilde{x}\| \geq \delta$. If $\|z-\tilde{x}\| \leq \delta$ we have

$$
|\Lambda(\tilde{x})| \leq(\|x-z\|+\|z-\tilde{x}\|)\|d s\|_{L^{\infty}}\|d \varphi\|_{L^{\infty}} \delta^{-3}+\|d s\|_{L^{\infty}}\|\varphi\|_{L^{\infty}} \delta^{-2} \leq C_{0} \delta^{-2}
$$

Since in addition $\left|\Gamma^{\prime} \cap B(z, \delta)\right| \leq C_{2} \delta$, we finally find that

$$
\left|\int_{\Gamma^{\prime}} \Lambda(\tilde{x})\langle\mathbf{n}(\tilde{x}), \mathbf{t}(x)\rangle d \tilde{x}\right| \leq C_{0} C_{1} C_{2}
$$

We have therefore proved that

$$
\left|\mathbf{t}(x)^{\mathrm{T}} I(z, x) \mathbf{t}(x)\right| \leq C,
$$

where the constant $C$ is independent of $\delta$, which shows that the contribution of $I(z, x)$ is negligible in (3.24).

We now analyze the contribution the quantity $[f] J(z)$ in (7.49). For this purpose, we use an expression of the second derivative of the characteristic function $\chi_{\Omega^{\prime}}$ of a smooth set $\Omega^{\prime}$ in the distribution sense. We assume without loss of generality that $\Gamma^{\prime}$ is parametrized in the trigonometric sense, and therefore that $\mathbf{n}$ is the inward normal to $\Omega$. For all test function $\psi$, we have

$$
-\int_{\Omega^{\prime}} \partial_{u, v}^{2} \psi=\int_{\Gamma^{\prime}} \partial_{u} \psi\langle v, \mathbf{n}\rangle=\int_{\Gamma^{\prime}}\left(\partial_{\mathbf{n}} \psi\langle u, \mathbf{n}\rangle+\partial_{\mathbf{t}} \psi\langle u, \mathbf{t}\rangle\right)\langle v, \mathbf{n}\rangle
$$

and, by integration by parts,

$$
\int_{\Gamma^{\prime}} \partial_{\mathbf{t}} \psi\langle u, \mathbf{t}\rangle\langle v, \mathbf{n}\rangle=-\int_{\Gamma^{\prime}} \psi(\langle u, \kappa \mathbf{n}\rangle\langle v, \mathbf{n}\rangle-\langle u, \mathbf{t}\rangle\langle v, \kappa \mathbf{t}\rangle) .
$$

Therefore, we have

$$
-\int_{\Omega^{\prime}} \partial_{u, v}^{2} \psi=\int_{\Gamma^{\prime}}\langle u, \mathbf{n}\rangle\langle v, \mathbf{n}\rangle\left(\partial_{\mathbf{n}} \psi-\kappa \psi\right)+\kappa\langle u, \mathbf{t}\rangle\langle v, \mathbf{t}\rangle \psi .
$$

Applying this formula to $\psi(\tilde{x}):=\varphi_{\delta}(z-\tilde{x})$ we obtain

$$
-u^{\mathrm{T}} J(z) v=\int_{\Gamma^{\prime}}\langle u, \mathbf{n}(\tilde{x})\rangle\langle v, \mathbf{n}(\tilde{x})\rangle\left(\partial_{\mathbf{n}} \varphi_{\delta}(z-\tilde{x})-\kappa(\tilde{x}) \varphi_{\delta}(z-\tilde{x})\right)+\kappa(\tilde{x})\langle u, \mathbf{t}(\tilde{x})\rangle\langle v, \mathbf{t}(\tilde{x})\rangle \varphi_{\delta}(z-\tilde{x}) d \tilde{x}
$$

Since $\Gamma_{j}$ is $C^{2}$, there exists a constant $C_{0}$ such that for all $x_{1}, x_{2} \in \Gamma_{j}$, we have

$$
\left|\left\langle\mathbf{t}\left(x_{1}\right), \mathbf{n}\left(x_{2}\right)\right\rangle\right| \leq C_{0}\left\|x_{1}-x_{2}\right\|,
$$

and

$$
\left|1-\left\langle\mathbf{n}\left(x_{1}\right), \mathbf{n}\left(x_{2}\right)\right\rangle\right|=\left|1-\left\langle\mathbf{t}\left(x_{1}\right), \mathbf{t}\left(x_{2}\right)\right\rangle\right| \leq C_{0}\left\|x_{1}-x_{2}\right\|^{2} .
$$

We finally remark that $\left|\Gamma^{\prime} \cap B(z, \delta)\right| \leq C_{1} \delta$, and that $\left\|\varphi_{\delta}\right\|_{L^{\infty}} \leq\|\varphi\|_{L^{\infty}} \delta^{-2}$ and $\left\|\partial_{\mathbf{n}} \varphi_{\delta}\right\|_{L^{\infty}} \leq\|d \varphi\|_{L^{\infty}} \delta^{-3}$.

Taking the vectors $\mathbf{t}(x)$ or $\mathbf{n}(x)$ as possible values of $u$ and $v$ in (7.53) and using the above remarks, we obtain the estimates

$$
\begin{aligned}
\left|\mathbf{n}(x)^{\mathrm{T}} J(z) \mathbf{n}(x)+\int_{\Gamma^{\prime}} \partial_{\mathbf{n}} \varphi_{\delta}(z-\tilde{x}) d \tilde{x}\right| & \leq C \delta^{-1}, \\
\left|\mathbf{t}(x)^{\mathrm{T}} J(z) \mathbf{n}(x)\right| & \leq C \delta^{-1}, \\
\left|\mathbf{t}(x)^{\mathrm{T}} J(z) \mathbf{t}(x)+\int_{\Gamma^{\prime}} \kappa(\tilde{x}) \varphi_{\delta}(z-\tilde{x}) d \tilde{x}\right| & \leq C,
\end{aligned}
$$


where the constant $C$ depends only on $f$. In view of (7.49) We can immediately derive estimate (3.23) from (7.55).

In order to derive the estimate (3.24) from (7.56), we first introduce the modulus $\omega$ of continuity of $\kappa$ on $\Gamma_{j}$,

$$
\omega(\delta):=\sup _{x_{1}, x_{2} \in \Gamma_{j} ;\left\|x_{1}-x_{2}\right\| \leq \delta}\left|\kappa\left(x_{1}\right)-\kappa\left(x_{2}\right)\right| .
$$

Therefore

$$
\left|\mathbf{t}(x)^{\mathrm{T}} J(z) \mathbf{t}(x)+\int_{\Gamma^{\prime}} \kappa(\tilde{x}) \varphi_{\delta}(z-\tilde{x}) d \tilde{x}\right| \leq\left|\mathbf{t}(x)^{\mathrm{T}} J(z) \mathbf{t}(x)+\kappa(x) \int_{\Gamma^{\prime}} \varphi_{\delta}(z-\tilde{x}) d \tilde{x}\right|+C \omega(\delta) \delta^{-1} .
$$

We now claim that

$$
\left|\int_{\Gamma} \varphi_{\delta}(z-\tilde{x}) d \tilde{x}-\delta^{-1} \Phi(u)\right| \leq C
$$

holds with $C$ independent of $\delta$ which implies the validity of (3.24). In order to prove (7.57), we use a local parametrization of $\Gamma^{\prime}$ : let $\lambda: \mathbb{R} \rightarrow \mathbb{R}$ be such that for $h$ small enough we have, $x+h \mathbf{t}(x)+\lambda(h) \mathbf{n}(x) \in \Gamma$. Note that we have $|\lambda(h)| \leq C_{0} h^{2}$ and $\left|\lambda^{\prime}(h)\right| \leq C_{0} h$ for $h$ small enough. Then for $\delta$ small enough,

$$
\begin{aligned}
\left|\int_{\Gamma^{\prime}} \varphi_{\delta}(z-\tilde{x}) d \tilde{x}-\delta^{-1} \Phi(u)\right| & \leq\left|\int_{\mathbb{R}} \varphi_{\delta}(h \mathbf{t}(x)+(\delta u-\lambda(h)) \mathbf{n}(x)) \sqrt{1+\lambda^{\prime 2}} d h-\int_{T_{x} \Gamma} \varphi_{\delta}(h \mathbf{t}(x)+\delta u \mathbf{n}(x)) d h\right| \\
& \leq C \delta\left(\left\|\varphi_{\delta}\right\|_{L^{\infty}}\left(\sqrt{1+\left(C_{0} \delta\right)^{2}}-1\right)+\left\|d \varphi_{\delta}\right\|_{L^{\infty}} C_{0} \delta^{2}\right) \leq C
\end{aligned}
$$

Finally, we can derive the estimate (3.22) from (7.54) using the inequality

$$
\left|\int_{\Gamma} \partial_{\mathbf{n}} \varphi_{\delta}(z-\tilde{x}) d \tilde{x}+\delta^{-2} \Phi^{\prime}(u)\right| \leq C \delta^{-1}
$$

which proof is very similar to the one of (7.57), using that $\left\|\mathbf{n}\left(x_{1}\right)-\mathbf{n}\left(x_{2}\right)\right\| \leq C\left\|x_{1}-x_{2}\right\|$ for all $x_{1}, x_{2} \in \Gamma_{j}$.

\section{References}

[1] F. Arandiga, A. Cohen, R. Donat, N. Dyn and B. Matei, Approximation of piecewise smooth images by edge-adapted techniques, Appl. Comp. Harm. Anal. 24, 225-250, 2008.

[2] V. Babenko, Y. Babenko, A. Ligun and A. Shumeiko, On Asymptotical Behavior of the Optimal Linear Spline Interpolation Error of $C^{2}$ Functions, East J. Approx. 12(1), 71-101, 2006.

[3] E. Candes and D. L. Donoho, Curvelets and curvilinear integrals, J. Approx. Theory. 113, 59-90, 2000.

[4] F. Cao, Geometric curve evolution and image processing, Lecture Notes in Mathematics, Springer, 2003.

[5] L. Chen, P. Sun and J. Xu, Optimal anisotropic meshes for minimizing interpolation error in L $^{p}$ norm, Math. of Comp. 76, 179-204, 2007.

[6] A. Cohen, Numerical analysis of wavelet methods, Elsevier, 2003.

[7] A. Cohen, W. Dahmen, I. Daubechies, and R. DeVore, Tree approximation and optimal encoding, Appl. Comp. Harm. Anal. 11, pp. 192-226, 2001.

[8] A. Cohen, R. DeVore, P. Petrushev, and H. Xu, Nonlinear approximation and the space BV $\left(\mathbb{R}^{2}\right)$, Amer. J. Math. 121, 587-628, 1999.

[9] R. DeVore, Nonlinear approximation, Acta Numerica, 7, 51-150, 1998.

[10] R. DeVore, G. Petrova and P. Wojtactzyck, Anisotropic Smoothness via Level Sets, Comm. Pure and Applied Math. 61, 1264-1297, 2008. 
[11] R. DeVore and B. Lucier, High order regularity for conservation laws, Indiana Math., 413-430, 1990.

[12] R. DeVore and G. Lorentz, Constructive approximation, Springer Grundlehren, Vol. 303, 1993.

[13] E. Fatemi, S. Osher and L. Rudin, Non linear total variation based noise removal algorithms, Physica D 60, 259-268, 1992

[14] E. Godlewski and P.A. Raviart Numerical approximation of hyperbolic systems of conservation laws, Applied Mathematical Sciences 118, Springer-Verlag, New-York, 1996.

[15] B. Karaivanov and P. Petrushev, Nonlinear piecewise polynomial approximation beyond Besov spaces, Appl. Comput. Harmon. Anal. 15, 177-223, 2003.

[16] E. Le Pennec and S. Mallat, Bandelet image approximation and compression, SIAM Journal of Multiscale Modeling. and Simulation 4, 992-1039, 2005.

[17] R. LeVeque, Numerical methods for conservation laws, Birkhauser, 1992.

[18] C. Louchet and L. Moisan, Total Variation denoising using posterior expectation, proceedings of the European Signal Processing Conference (Eusipco), 2008

[19] J.M. Mirebeau, Optimal meshes for finite elements of higher order, to appear in Constructive Approximation, 2010.

[20] S.M. Nikol'skij, Approximation of functions of several variables and imbedding theorems, Springer, 1975.

[21] P. J. Olver, G. Sapiro and A. Tannenbaum, Invariant geometric evolutions of surfaces and volumetric smoothing, SIAM J. Appl. Math. 57,176-194, 1997.

[22] H. Whitney, Analytic extensions of differentiable functions defined in closed sets, Transactions of the American Mathematical Society, 36, 63-89, 1934.

[23] V. Temlyakov, Approximation of periodic functions, Nova Science, New York, 1993.

Albert Cohen

UPMC Univ Paris 06, UMR 7598, Laboratoire Jacques-Louis Lions, F-75005, Paris, France CNRS, UMR 7598, Laboratoire Jacques-Louis Lions, F-75005, Paris, France cohen@ann.jussieu.fr

Jean-Marie Mirebeau

UPMC Univ Paris 06, UMR 7598, Laboratoire Jacques-Louis Lions, F-75005, Paris, France CNRS, UMR 7598, Laboratoire Jacques-Louis Lions, F-75005, Paris, France

mirebeau@ann.jussieu.fr 